\title{
A FAMILY OF SIMPLE GROUPS ASSOCIATED WITH THE SATAKE DIAGRAMS
}

\author{
CHENG CHONHU \\ (Received 8 September 1983; revised 13 October 1984) \\ Communicated by D. E. Taylor
}

\begin{abstract}
Using the theory of the Satake diagrams associated with the non-compact simple Lie algebras over the real number field $\mathbf{R}$, we shall construct a family of simple groups over a field $K$ which are called the simple groups associated with the Satake diagrams. The list of these simple groups includes all Chevalley groups and twisted groups, and all simple algebraic groups of adjoint type defined over $\boldsymbol{R}$ if $K$ is the complex number field $\mathbf{C}$ (except two types given by Table $\mathrm{II}^{\prime}$ ). Furthermore, the simple groups associated with the Satake diagrams of type AIII, BI, DI are identified with the simple groups obtained from the unitary or orthogonal groups of non-zero indices. The quasi-Bruhat decomposition of the "non-split" simple groups associated with the Satake diagrams which are not Chevalley groups or twisted groups will be given in this paper.
\end{abstract}

1980 Mathematics subject classification (Amer. Math. Soc.): $20 \mathrm{H} 20$.

\section{Introduction}

Every Satake diagram $\Pi^{\theta}=(\Pi, \theta)$ associated with a non-compact simple Lie algebra $g$ over $\mathbf{R}$ determines an involutive automorphism $\rho_{\theta}$ of $L$ where $L$ is a simple Lie algebra over $\mathbf{C}$ which is defined by $L=g+\sqrt{-1} g$. Using this involutive automorphism $\rho_{\theta}$ of $L$ and an involutive automorphism $f$ of a field $K$ we shall construct a simple group over $K$ which will be called a simple group associated with the Satake diagram $\Pi^{\theta}$ over $K$ and will be denoted by $L\left(\Pi^{\theta}, K\right)$ (or $L(\Pi, \theta ; K, f)$ ). The procedures of the construction of such simple groups $L\left(\Pi^{\theta}, K\right)$ are quite analogous to those in [3], [6], [2]. The list of these simple groups $L\left(\Pi^{\theta}, K\right)$ includes all Chevalley groups and twisted groups over $K$. In general, the classical groups which are Chevalley groups or twisted groups are the

(ㄷ) 1986 Australian Mathematical Society $0263-6115 / 86 \$ A 2.00+0.00$ 
linear and symplectic groups, and the orthogonal and unitary groups corresponding to forms whose Witt index is sufficiently large. The remaining classical groups are not Chevalley groups or twisted groups. Furthermore, the simple groups associated with the Satake diagram of the type AIII, BI, DI are identified with the simple groups obtained from unitary or orthogonal groups of non-zero indices. The Chevalley groups and twisted groups can be interpreted as "split" groups of Lie type, the remaining simple groups $L\left(\Pi^{\theta}, K\right)$ can be interpreted as "non-split" groups of Lie type (cf. [1], page 216). The quasi-Bruhat decomposition of such "non split" simple groups will be given in this paper (cf. Theorem 1)

The classification of semi-simple algebraic groups defined over $\mathbb{R}$ is considered by $J$. Tits [7] and by I. Satake [5]. The problem of classifying the semi-simple algebraic groups defined over $\boldsymbol{R}$ is reduced to the problem of finding the admissible $(\mathbb{R})$-indices of semi-simple algebraic groups defined over $\mathbb{R}$ and all possible semi-simple anisotropic kernels for a given admissible $(\mathbb{R})$-index. J. Tits [7] enumerated all possible admissible $(\mathbb{R})$-indices which are all Satake diagrams.

For each admissible ( $\mathbb{R}$ )-index of type $A_{l}, B_{l}, C_{l}, D_{l} \mathrm{~J}$. Tits [7] gave a classical group with identifies the simple algebraic group defined over $\mathbb{R}$ with such index. For each admissible ( $(\mathbb{R})$-index of type $G_{2}, F_{4}, E_{7}, E_{8}$ (except certain indices, for example, the index $E V I$, i.e., $E_{7,4}^{9}$ in [7]), J. Tits [7] constructed the simple algebraic group defined over $\mathbb{R}$ with such index by means of Cayley algebra, Jordan algebra, associative division algebra, and division Cayley algebra.

For all Satake diagrams $(\Pi, \theta)$ the construction of the groups $L(\Pi, \theta ; \mathbf{C}, f)$ given by our paper is just a uniform construction of simple algebraic groups of adjoint type defined over $\boldsymbol{R}$. Thus for all possible admissible $(\mathbb{R})$-indices a uniform proof of the existence of simple algebraic groups of adjoint type defined over $\mathbb{R}$ is given in our paper.

In this paper, we shall use the notations and terminology defined in [1] and [4] without explanation and we shall not consider two Satake diagrams given by Table II'.

We shall denote by $\Pi^{\theta}$ the Satake diagram $(B, \theta)$ defined by [4] which corresponds to a noncompact simple Lie algebra $g$ over $\mathbb{R}$. Let $g=t+p$ be a Cartan decomposition of $g$ and $\theta$ be the Cartan involution of $g$ [4]. Clearly, the Cartan involution $\theta$ can be extended to an automorphism of $L$ which is denoted by $\theta$ also in the following way:

$$
\theta(X+\sqrt{-1} Y)=\theta X+\sqrt{-1} \theta Y, \quad X, Y \in g .
$$

Let $a$ be a maximal abelian subalgebra of $p$. Then there exists a Cartan subalgebra $h$ of $g$ such that $h=h_{t}+\mathfrak{a}, h_{t} \subset t$ [4]. We denote by $\Phi$ the root system of $L$ with respect to the Cartan subalgebra $h^{c}=h+\sqrt{-1} h$ of $L$ with the fundamental root system $B$ (denoted by $\Pi$ in this paper). For each $r \in \Phi$ we define $\bar{r}=-\theta(r)$, so $r^{\prime}=\frac{1}{2}(r+\bar{r})$ is the restriction of $r$ to $a$. We can define an 
ordering on $a$ which is compatible with the ordering on the space $\Gamma$ spanned by $\Phi$ over $\mathbb{R}$.

Let $\tilde{C}_{b}:\left\{h_{r}, r \in \Pi ; \tilde{e}_{r^{\prime}}, r \in \Phi\right\}$ be a Chevalley basis of $L$ and $\tau$ be the non-trivial symmetry of the Dynkin diagram $\Pi$ which is given by 13.6 .2 of [1]. For each $r \in \Phi$ we define $\tilde{x}_{r}(t)=\exp \left(t \operatorname{ad} \tilde{e}_{r}\right), \quad t \in K, \quad \tilde{e}_{r} \in \tilde{C}_{b}, \tilde{n}_{r}(t)=$ $\tilde{x}_{r}(t) \tilde{x}_{-r}\left(-t^{-1}\right) \tilde{x}_{r}(t), t \in K^{*}$, and $\tilde{h}_{r}(t)=\tilde{n}_{r}(t) \tilde{n}_{r}(-1)$. We denote by $I$ the identical map of $L$ and by $\varnothing$ the empty set.

We define $\Pi^{0}=\{\alpha \in \Pi \mid \alpha=-\bar{\alpha}\}$ and $\Phi^{0}=\{\alpha \in \Phi \mid \alpha=-\bar{\alpha}\}$. Clearly $\Pi^{0}$ is a subset of II consisting of all simple roots which correspond to the black nodes in the Satake diagram $(\Pi, \theta)$ and $\Phi^{0}$ is a root system with the fundamental system $\Pi^{0}$. We denote by $\Pi^{*}$ the set $\Pi \backslash \Pi^{0}$ and by $\Phi^{*}$ the set $\Phi \backslash \Phi^{0}$. Furthermore, we define $\Phi_{1}=\left\{r \in \Phi^{*} \mid r=\bar{r}\right\}, \Phi_{11}=\Phi^{*} \backslash \Phi_{1}, \quad \Phi_{11 b}=\{r \in$ $\left.\Phi_{11} \mid r+\bar{r} \in \Phi\right\}, \quad \Phi_{11 a}=\Phi_{11} \backslash \Phi_{11 b}, \quad \Phi_{1 b}=\left\{r+\bar{r} \mid r \in \Phi_{11 b}\right\}$ and $\Phi_{1 a}=$ $\Phi_{1} \backslash \Phi_{1 b}$.

Proposition 1. For each Satake diagram $(\Pi, \theta)$ there exist a Chevalley basis $C_{b}=\left\{h_{r}, r \in \Pi ; e_{r}, r \in \Phi\right\}$ of $L$ and an involutive automorphism $\rho_{\theta}$ of $L$ such that $\rho_{\theta}\left(e_{r}\right)=k_{r} e_{\bar{r}}, r \in \Phi, e_{r}, e_{\bar{r}} \in C_{b}, k_{r}= \pm 1$ satisfying the condition

$$
k_{r}=1 \quad \text { if } r \in \Phi_{11} \cup \Phi_{1 a}, \quad k=-1 \quad \text { if } r \in \Phi_{1 b} \text {. }
$$

Proof. We define $\Pi_{0}^{*}=\left\{r \in \Pi^{*} \mid(r, \alpha)=0\right.$ for all $\left.\alpha \in \Pi^{0}\right\}$. For each $r \in$ $\Pi^{*} \backslash \Pi_{0}^{*}$ if there eixsts $s \in \Pi^{*} \backslash \Pi_{0}^{*}$ such that $s^{\prime}=r^{\prime}$ and $r \neq s$ then $r, s$ is called a simple root of type II, otherwise $r$ is called a simple root of type I. It follows from the properties of the Satake diagrams that $\bar{r}=r+\beta, \beta=\Sigma \beta_{i}$, $\beta_{i} \in \Pi^{0}$ if $r$ is a simple root of type $\mathrm{I}, \bar{r}=s+\beta, \bar{s}=r+\beta, \beta \in \Phi^{0}$ if $r, s$ is a simple root of type II (cf. Table $\mathrm{I}_{4}$ ). There is an automorphism $\theta_{0}$ of $L$ such that $\theta_{0}\left(h_{r}\right)=-h_{r}, \theta_{0}\left(\tilde{e}_{r}\right)=-\tilde{e}_{-r}, r \in \Phi, \tilde{e}_{r}, \tilde{e}_{-r} \in \tilde{C}_{b}$ (cf. [1], page 56). We define an automorphism $\rho$ of $L$ by $\rho=\theta_{0} \theta$. Let $\bar{X}$ denote $\rho X$ for each $X \in h^{c}$. It is clear that there exists $\tilde{X} \in h^{c}$ such that the automorphism $\tilde{\rho}$ of $L$ defined by $\tilde{\rho}=$ $\rho \exp (\operatorname{ad} \tilde{X})$ satisfies $\tilde{\rho}\left(\tilde{e}_{r}\right)=\eta_{r} \tilde{e}_{\tilde{r}}, r \in \Phi, \tilde{e}_{r}, \tilde{e}_{\tilde{r}} \in \tilde{C}_{b}, \eta_{r} \in \mathbf{C}$ satisfying $\eta_{r}=1$ for all $r \in \Pi_{0}^{*}$ and $\eta_{\alpha}=-1$ for all $\alpha \in \Pi^{0}$. Since $\overline{\bar{r}}=r$ for all $r \in \Phi$ we have $\tilde{\rho}^{2}=\exp \left(\operatorname{ad} X_{0}\right), X_{0} \in h^{c}$. It follows that $\eta_{r} \eta_{\bar{r}}=e^{\left(X_{0}, r\right)}$ and $\eta_{r} \eta_{\bar{r}}=e^{\left(X_{0}, \bar{r}\right)}$ for all $r \in \phi$, so we have $\left(X_{0}, r\right)=\left(X_{0}, \bar{r}\right)(\bmod 2 \pi \sqrt{-1}), r \in \phi$. Thus we have $\left(X_{0}, r\right)$ $\equiv 0(\bmod 2 \pi \sqrt{-1}), r \in \Pi^{0} \cup \Pi_{0}^{*},\left(X_{0}, r\right)=\left(X_{0}, \bar{r}\right)(\bmod 2 \pi \sqrt{-1}), r \in \Pi^{*} \backslash \Pi_{0}^{*}$. Clearly, there exists $X^{*} \in h^{c}$ such that $X^{*}$ satisfies the relation $\left(X^{*}, r\right)=0$, $r \in \Pi^{0} \cup \Pi_{0}^{\prime},\left(X^{*}, r\right)=\left(X_{0}, r\right), r \in \Pi^{*} \backslash \Pi_{0}^{*}$. Since $\tilde{r} \in \pm\left(\Pi^{0} \cup \Pi_{0}^{*}\right)$ for all $r \in \Pi^{0} \cup \Pi_{0}^{*}$ we have $\left(\bar{X}^{*}, r\right)=0, r \in \Pi^{0} \cup \Pi_{0}^{*}$ and $\left(X^{*}, \beta\right)=\left(\bar{X}^{*}, \beta\right)=0$, $\beta \in \phi^{0}$. By the formula mentioned above we have $\left(X^{*}, r\right)=\left(\bar{X}^{*}, \bar{r}\right)=(\bar{X}, r+$ $\beta)=\left(\bar{X}^{*}, r\right), \beta=\sum \beta_{i}, \beta \in \Pi^{0}$ if $r$ is of type $\mathrm{I},\left(X^{*}, r\right)=\left(X_{0}, r\right)=\left(X_{0}, \bar{s}-\beta\right)$ $\equiv\left(X_{0}, \bar{s}\right) \equiv\left(X_{0}, s\right)=\left(X^{*}, s\right)=\left(X^{*}, \bar{r}-\beta\right)=\left(X^{*}, \bar{r}\right)=\left(\bar{X}^{*}, r\right)(\bmod 2 \pi \sqrt{-1})$ 
if $r, s$ is of type II. We define $\rho_{\theta}=\tilde{\rho} \exp \left(\operatorname{ad}\left(-\frac{1}{2} X^{*}\right)\right)$. We have

$$
\rho_{\theta}^{2}=\tilde{\rho}^{2} \exp \left(\operatorname{ad}\left(-\frac{1}{2}\left(X^{*}+\bar{X}^{*}\right)\right)\right)=\exp \left(\operatorname{ad}\left(X_{0}-\frac{1}{2}\left(X^{*}+\bar{X}^{*}\right)\right)\right),
$$

so we have $\rho_{\theta}^{2}\left(\tilde{e}_{r}\right)=\tilde{e}_{r}, \tilde{e}_{r} \in \tilde{C}_{b}$, for all $r \in \Pi$. Thus $\rho_{\theta}$ is an involutive automorphism of $L$ and $\rho_{\theta}\left(\tilde{e}_{r}\right)=\tilde{e}_{\tilde{r}}, r \in \Pi_{0}^{*}$, since $\left(X^{*}, r\right)=0$ and $\eta_{r}=1$ for all $r \in \Pi_{0}^{*}$. Clearly, $\Phi_{1 a} \cap \Pi_{0}^{*}$ is a fundamental root system $\Phi_{1 a}$, so $\rho_{\theta}\left(\tilde{e}_{r}\right)=\tilde{e}_{r}$, $r \in \Phi_{1 a}$. Another proof of the existence of $\rho_{\theta}$ will be given below. For each $\Pi^{\theta}$ we define $A_{\theta}=\left(\tau_{\theta} ; \Psi_{\theta} ; \varphi_{\theta}\right)$ where $\tau_{\theta}=I$ or $\tau$, and $\Psi_{\theta}=\left\{\beta_{1}, \beta_{2}, \ldots, \beta_{p}\right\}$ and $\varphi_{\theta}=$ $\left\{\delta_{1}, \delta_{2}, \ldots, \delta_{q}\right\}$ are the subsets of $\Phi$ determined in individual cases by inspecting the Satake diagram $\Pi^{\theta}$. For each Satake diagram $\Pi^{\theta}, A_{\theta}$ is given in Table II. Furthermore, for each $A_{\theta}$ we define $\rho_{\theta}=\tau_{\theta} h_{\theta} n_{\theta}$ where $h_{\theta}=h_{\beta_{1}}(-1) h_{\beta_{2}}(-1) \cdots$ $h_{\beta_{p}}(-1)$ if $\Psi_{\theta} \neq \varnothing, h_{\theta}=I$ if $\Psi_{\theta}=\varnothing$ and $n_{\theta}=n_{\delta_{1}}(1) n_{\delta_{2}}(1) \cdots n_{\delta_{q}}(1)$ if $\varphi_{\theta} \neq \varnothing$, $n_{\theta}=I$ if $\varphi_{\theta}=\varnothing$. It is easily verified that $\rho_{\theta}$ is an involutive automorphism of $L$ and $\rho_{\theta} e_{r}=c_{r} e_{r}$ for all $r \in \Phi, c_{r}= \pm 1$, satisfying $c_{r}=1$ for all $r \in \Phi_{1 a}$. We shall show that we can choose an appropriate $\xi_{r}, \xi_{r}= \pm 1$ such that $\rho_{\theta}\left(e_{r}\right)=k_{r} e_{r}$, $r \in \Phi, e_{r}=\xi_{r} \tilde{e}_{r}, \tilde{e}_{r} \in \tilde{C}_{b}, k_{r}= \pm 1$, satisfying the condition (A). Since $\rho_{\theta}^{2}=1$ we have

$$
k_{r} k_{-r}=1, \quad k_{r} k_{\tilde{r}}=1, \quad k_{r} k_{s} N_{\tilde{r}, \bar{s}}=N_{r, s} k_{r+s}, \quad r, s \in \Phi .
$$

We put $\xi_{r}=1$ for all $r \in \Phi_{1 a} \cup \Pi$. Without loss of generality, we assume $h(r) \leqslant h(\bar{r}), r \in \Phi_{11}$ where $h(r)$ and $h(\bar{r})$ are the height of $r$ and $\bar{r}$ respectively, and then we put $\xi_{r}=1$ for all such $r$ of $\Phi_{11}$. It is clear that we can choose $\xi_{\vec{r}}$ such that $k_{r}=1$, so $k_{\dot{r}}=1$ by (B). If $r, s \in \Phi$ and $r+s \in \Phi$ then $N_{r, s}=-N_{s, r}$. Thus we have $k_{r}=-1$ for all $r \in \Phi_{1 b}$ by (B). Since $\xi_{r}=1$ for all $r \in \Pi$ then $C_{b}\left\{h_{r}, r \in \Pi ; e_{r}, r \in \Phi\right\}$ is a Chevalley basis of $L$ by page 56 of [1]. The proof is complete.

Since $\Pi_{0}^{*}=\left\{r \in \Pi^{*} \mid(r, \alpha)=0\right.$ for all $\left.\alpha \in \Pi^{0}\right\}$, we have the following statements:

(0) $\rho_{\theta}(\alpha)=-\alpha$ for all $\alpha \in \Pi^{0}$ means $\rho_{\theta}$ transforms every root $\alpha$ corresponding to the black circle of $\Pi^{\theta}$ to $-\alpha$.

(I) $\rho_{\theta}(r)=r, r \in \Pi_{0}^{*}$ means $\rho_{\theta}$ transforms the circle corresponding to $r$ to itself, this circle being white and being not joined by any arrow to any circle in $\Pi^{\theta}$.

(II) $\rho_{\theta}(r)=s, r, s \in \Pi_{0}^{*}, r \neq s$ means $\rho_{\theta}$ transforms the circle corresponding to $r$ to the circle corresponding to $s$, these two circles being white and being joined by an arrow in $\Pi^{\theta}$.

In this paper, we denote by $K$ a field of characteristic $p>3$ (if the dimension of $a$ is equal to $1, K$ is a field of characteristic $p>5$ ). Let $f$ be a non-trivial involutive automorphism of $K$. For each $t \in K$ we write $f(t)=\bar{t}$. Clearly, the Chevalley group $G=L(K)$ of type $L$ over $K$ is generated by $\left\{x_{r}(t)=\right.$ $\left.\exp \left(t \operatorname{ad} e_{r}\right), r \in \Phi, t \in K\right\}$. Obviously, the involutive automorphism $f$ of $K$ can 
be extended to an involutive automorphism of $G$ (denoted by $f$ also) in this way: $f\left(x_{r}(t)\right)=x_{r}(\bar{t})$ for all $r \in \Phi, t \in K$. The involutive automorphism $\rho_{\theta}$ of $L$ can be extended to an involutive automorphism of $G$ (denoted by $\rho_{\theta}$ also) in this way: $\rho_{\theta}\left(x_{r}(t)\right)=x_{\vec{r}}\left(k_{r} t\right)$ for all $r \in \Phi, t \in K$. We define an automorphism $\sigma$ of $G$ by $\sigma=\phi_{\theta} f$, so $\sigma\left(x_{r}(t)\right)=x_{\bar{r}}\left(k_{r} \bar{t}\right), r \in \Phi, t \in K$. Since $\rho_{\theta} f=f \rho_{\theta}$, and $\rho_{\theta}^{2}=I$ and $f^{2}=I$ we have $\sigma^{2}=I$.

\section{The subgroups of $L\left(\Pi^{\theta}, K\right)$}

We define some notation and terminology which will be used later.

I. If $r \in \Phi_{1}, X_{r}^{1}(t)=x_{r}(t), t \in K_{r}=\left\{t \in K \mid t=k_{r} \bar{t}\right\}, W_{r}^{1}=w_{r}, N_{r}^{1}(u)=$ $n_{r}(u), u \in K_{r}^{*}=K_{r} \backslash\{0\}, h_{r}^{1}(v)=h_{r}(v), v \in K_{r}^{*}=\{v \in K \mid v=\ddot{v}\} \backslash\{0\}$.

IIa. If $r \in \Phi_{11 a}, \quad X_{r}^{1}(t)=x_{r}(t) x_{\bar{r}}(\bar{t}), \quad t \in K_{r}=K, \quad W_{r}^{1}=w_{r} w_{\bar{r}}, \quad N_{r}^{1}(u)=$ $n_{r}(u) n_{\bar{r}}(\bar{u}), u \in K_{r}^{*}=K \backslash\{0\}, h_{r}^{1}(v)=h_{r}(v) h_{\bar{r}}(\bar{v}), v \in K_{r}^{* \prime}=K^{*}=K \backslash\{0\}$.

IIb. If $r \in \Phi_{11 b}, X_{r}^{1}(t)=x_{r}(t) x_{\bar{r}}(\bar{t}) x_{r+\bar{r}}\left(-\frac{1}{2} N_{r, \bar{r}} t \bar{t}\right), t \in K_{r}=K, W_{r}^{1}=w_{r+\bar{r}}$, $N_{r}^{1}(u)=n_{r+\bar{r}}(u), u \in K_{r+\bar{r}}, h_{r}^{1}(v)=h_{r}(v) h_{\bar{r}}(\bar{v}), v \in K_{r}^{* \prime}=K^{*}$.

For each $r \in \Phi^{*}$ we define $I(r)=\left\{s \in \Phi^{*} \mid s^{\prime}=k r^{\prime}, k=1,2\right.$, or $\left.\frac{1}{2}\right\}$ and $I_{1}(r)=\{s \in I(r) \mid s \leqslant \bar{s}\}$. If $I_{1}(r)=\left\{r_{1}, r_{2}, \ldots, r_{n}\right\}, r_{1}<r_{2}<\cdots<r_{n}, r_{i} \in$ $\Phi^{*}, i=1,2, \ldots, n$, we define $X_{r}(T)=\prod_{i=1}^{n} X_{r_{i}}\left(t_{i}\right), t_{i} \in K_{r_{i}}, T=\left(t_{1}, t_{2}, \ldots, t_{n}\right)$ and $D(T)=t_{1} \bar{t}_{1}+t_{2} \bar{t}_{2}+\cdots+t_{n} \bar{t}_{n}$. We write $N_{r}^{1}=N_{r}^{1}(1)$ if $\quad r \in \Phi_{1 a} \cup \Phi_{11 a}$, $N_{r}^{1}=h_{r}^{1}(u) n_{r}, u$ being a fixed element of $K_{r}^{*}$ if $r \in \Phi_{1 b}$.

We denote by $\Phi^{+}$(respectively $\Phi^{-}$) the positive (negative) root system containing $\Pi(-\Pi)$. Furthermore, we define $W^{1}=\left\langle W_{r}^{1}, r \in \Phi^{*}\right\rangle, N^{1}=\left\langle N_{r}^{1}(u), r \in\right.$ $\left.\Phi^{*}, u \in K_{r}^{*}\right\rangle$ and $H^{1}=N^{1} \cap H$. We define $U^{1}=\{u \in U \mid \sigma u=u\}, V^{1}=\{v$ $\in V \mid \sigma v=v\}$ and $G^{1}=\left\langle U^{1}, V^{1}\right\rangle$. Clearly, $G^{1}$ is determined by the Satake diagram $\Pi^{\theta}=(\Pi, \theta)$ and $K, f$, so we denote by $L(\Pi, \theta ; K, f)$ (or $\left.\left(\Pi^{\theta} ; K\right)\right)$ the group $G^{\mathbf{1}}$.

Clearly, we have $\Gamma=\Gamma_{+}+\Gamma_{-}$where $\Gamma_{ \pm}=\left\{\gamma \in \Gamma \mid \rho_{\theta}(\gamma)= \pm \gamma\right\}$ so we have $\Gamma_{+}=\mathfrak{a}$ and $\Gamma_{-}=\sqrt{-1} h_{t}$. We define $W_{0}=\left\langle w_{\alpha}, \alpha \in \Phi^{0}\right\rangle$ and $W_{0}^{1}=W_{0} \cap W^{1}$. For each $r \in \Phi, r^{\prime}=\frac{1}{2}(r+\bar{r})$ is the restriction of $r$ to $\Gamma_{+}$.

\section{Proposition 2.}

(a) Let $w \in W^{1}$ satisfy $w(\gamma)=\gamma$ for all $\gamma \in \Gamma_{+}$. Then $w \in W_{0}^{1}$.

(b) Let $r_{1}, s \in \Phi^{*}$. Then there exists $w \in W^{1}$ such that $\left(s_{1}^{\prime}, r_{1}^{\prime}\right) \neq 0, s_{1}=w(s)$.

(c) Let $s \in \Phi^{*}$. Then there exist $w \in W^{1}$ and $r \in \Pi^{*}$ such that $w(s) \in I(r)$.

Proof. (a) Since $w \in W^{1}$ we have $\rho_{\theta}^{\prime} w=w \rho_{\theta}^{\prime}$ where $\rho_{\theta}^{\prime}$ is the restriction of $\rho_{\theta}$ to $\Gamma$, so we have $w\left(\Gamma_{-}\right)=\Gamma_{-}$. Thus the restriction of $w$ to $\Gamma_{-}$which is denoted by $\tilde{w}$ can be expressed in the form $\tilde{w}=t_{0} w_{1}$ where $t_{0}$ is a symmetry of $\Pi^{0}$ and $w_{1} \in W_{0}=W\left(\Phi^{0}\right)$. It is clear that $w_{1}^{-1} w(\gamma)=\gamma$ for all $\gamma \in \Gamma_{+}$since $\Gamma_{-}$is 
spanned by $\Phi^{0}$ over $\mathbb{R}$ and $\left(\Gamma_{+}, \Gamma_{-}\right)=0$. Obviously, we have $w_{1}^{-1} w\left(\Pi^{0}\right)=\Pi^{0}$. Since the ordering of $\Gamma_{+}$is compatible with the ordering of $\Gamma$ we have $w_{1}^{-1} w\left(\Phi^{+}\right)=\Phi^{+}$so $w e$ have $w_{1}^{-1} w=1$. It follows that $w=w_{1} \in W_{0} \cap W^{1}=W_{0}^{1}$.

(b) Let $\tilde{\Phi}=\Phi^{*} \backslash \Phi_{11 b}$ and $\tilde{\Phi}^{\prime}$ be the restriction of $\tilde{\Phi}$ to $\Gamma_{+}$. Clearly, $\tilde{\Phi}^{\prime}$ is a root system and there is a fundamental root system $\tilde{\Pi}^{\prime}$ of $\tilde{\Phi}^{\prime}$ such that $\tilde{\Pi}^{\prime} \subset \Phi^{+\prime}$ where $\Phi^{+\prime}$ is the restriction of $\Phi^{+} \cap \Phi^{*}$ to $\Gamma_{+}$. If $r \in \tilde{\Phi}$ then either $r=\bar{r}$ or $r \neq \bar{r}$ having $(r, \bar{r})=0$, so we have

$$
w_{r^{\prime}} s^{\prime}=s^{\prime}-\frac{2\left(s^{\prime}, r^{\prime}\right)}{\left(r^{\prime}, r^{\prime}\right)} r^{\prime}=s^{\prime}-\frac{2(s, r+\bar{r})}{(r+\bar{r}, r+\bar{r})}(r+\bar{r})=\left(w_{r}^{1}(s)\right)^{\prime}, \quad s \in \Phi .
$$

It follows that $W\left(\tilde{\Phi}^{\prime}\right)=W(\tilde{\Phi})^{\prime}$ where $W(\tilde{\Phi})^{\prime}$ is the restriction of $W(\tilde{\Phi})$ to $\Gamma_{+}$. There exists $\tilde{s}^{\prime}, \tilde{r}_{1}^{\prime} \in \tilde{\Phi}^{\prime}$ such that $\tilde{s}^{\prime}=k s^{\prime}, k=1$ or 2 and $\tilde{r}_{1}^{\prime}=k^{\prime} r_{1}^{\prime}, k^{\prime}=1$ or 2 . It is clear that there is $w^{\prime} \in W\left(\tilde{\Phi}^{\prime}\right)$ such that $\left(w^{\prime}\left(\tilde{s}^{\prime}\right), \tilde{r}_{1}^{\prime}\right) \neq 0$. By $(\mathrm{C})$, there is $w \in W(\tilde{\Phi}) \subset W^{1}$ such that $k k^{\prime}\left((w(s))^{\prime}, r_{1}^{\prime}\right) \neq 0$.

(c) There is $\tilde{s}^{\prime} \in \tilde{\Phi}$ such that $\tilde{s}^{\prime}=k s^{\prime}, k=1$ or 2 . Clearly, there is $\tilde{r}^{\prime} \in \tilde{\Pi}^{\prime}$ and $w^{\prime} \in W\left(\tilde{\Phi}^{\prime}\right)$ such that $w^{\prime}\left(\tilde{s}^{\prime}\right)=\tilde{r}^{\prime}$. There is $r \in \Pi^{*}$ such that $\tilde{r}^{\prime}=k^{\prime} r^{\prime}, k^{\prime}=1$ or 2. By (C), there is $w \in W(\tilde{\Phi}) \subset W^{1}$ such that $k^{\prime} r^{\prime}=\tilde{r}^{\prime}=w^{\prime}\left(\tilde{s}^{\prime}\right)=k w^{\prime}\left(s^{\prime}\right)=$ $k(w(s))^{\prime}$, so we have $w(s) \in I(r), r \in \Pi^{*}$. The proof is complete.

We define $\Phi_{1}^{*+}=\left\{r \in \Phi^{*+} \mid r \leqslant \bar{r}\right\}$ where $\Phi^{*+}=\Phi^{*} \cap \Phi^{+}$. Furthermore, we define $\Sigma_{1}^{*+}=\left\{r \in \Phi_{1}^{*+} \mid r \leqslant s\right.$ for all $\left.s \in I(r)\right\}$. Let $\Phi_{1}^{*}=\Phi_{1}^{*+} \cup-\Phi^{*-}$ and $\Sigma^{*}=\Sigma_{1}^{*+} \cup-\Sigma_{1}^{*+}$. If $r \in \Sigma_{1}^{*+}, I_{1}(r)=\left\{r=r_{1}, r_{2}, \ldots, r_{n}\right\}, r_{1}<r_{2}<\cdots<$ $r_{n}, r_{i} \in \Phi_{1}^{*+}, i=1,2, \ldots, n$, we write $K_{(r)}=K_{r_{1}} \times K_{r_{2}} \times \cdots \times K_{r_{n}}$. Assuming $r_{1} \in \Pi^{*}, r_{1} \in \Sigma_{1}^{*+}$ if $r_{1} \notin \Phi_{11 b}, r_{1} \notin \Sigma_{1}^{*+}$ if $r_{1} \in \Phi_{11 b}$ and there is $r \in I\left(r_{1}\right) \cap$ $\Sigma_{1}^{*+}, r_{i} \neq r$.

We shall omit the proof of the following Proposition 3 which is similar to those of 13.6.1 and 13.6.4 of [1], and 2.8 and 2.10 of [2].

PROPOSITION 3.

(a) Let $r \in \Phi_{1}^{*+}, s \in \Sigma_{1}^{*+}, t \in K_{r}$ and $T \in K_{(s)}$. Then $X_{r}^{1}(t), X_{s}(T) \in U^{1}$ and $X_{-r}^{1}(t), X_{-s}(T) \in V^{1}$.

(b) Each element $u$ of $U^{1}(u \neq 1)$ and each element $v$ of $V^{1}(v \neq 1)$ have a unique expression in the forms:

$$
\begin{gathered}
u=\prod X_{r_{i}}^{1}\left(t_{i}\right)=\prod X_{s_{j}}\left(T_{j}\right), \quad r_{i} \in \Phi_{1}^{*+}, s_{j} \in \Sigma_{1}^{*+}, t_{i} \in K_{r_{i}}^{*}, T_{j} \in K_{\left(s_{j}\right)}, \\
i=1,2, \ldots, p, j=1,2, \ldots, q, r_{1}<r_{1}<\cdots<r_{p}, s_{1}<s_{1}<\cdots<s_{q} . \\
v=\prod X_{-\gamma_{i}}^{1}\left(t_{i}\right)=\prod X_{-\delta_{j}}\left(T_{j}\right), \quad \gamma_{i} \in \Phi_{1}^{*+}, \delta_{j} \in \Sigma_{1}^{*+}, t_{i} \in K_{\gamma_{i}}^{*}, T_{j} \in K_{\left(\delta_{j}\right)}, \\
i=1,2, \ldots, l, j=1,2, \ldots, m, \gamma_{1}<\gamma_{2}<\cdots<\gamma_{l}, \delta<\delta_{2}<\cdots<\delta_{m} .
\end{gathered}
$$


Let $J_{1}(u)$ and $J_{1}(v)$ denote $r_{1}$ and $-\gamma_{1}$ respectively. We shall denote by $J(u)$ the set $\left\{r_{1}, r_{2}, \ldots, r_{p}\right\}$ and by $J(v)$ the set $\left\{-\gamma_{1},-\gamma_{2}, \ldots,-\gamma_{l}\right\}$.

\section{PROPOSITION 4.}

(a) Let $n \in N^{1}, h \in H^{1}$ and $w \in W^{1}$. Then $\sigma n=n$, $\sigma h=h$ and $\rho_{\theta}^{\prime} w=w \rho_{\theta}^{\prime}$

(b) $H^{1} \subset N^{1} \subset G^{1}$.

(c) $N^{1} / H^{1} \cong W^{1}$.

(d) $W^{1}=\left\langle W_{r}^{1}, r \in \Pi^{*}, W_{0}^{1}\right\rangle$.

Proof. It is easy to see that $r+\bar{r} \notin \Phi$ for each $r \in \Phi_{11 a}$, so for each $u \in K_{r}^{*}$, $N_{r}^{1}(u)$ can be expressed in the form $N_{r}^{1}(u)=X_{r}^{1}(u) X_{-r}^{1}\left(-u^{-1}\right) X_{r}^{1}(u)$ and hence $N_{r}^{1}(u) \in G^{1}$. The other results of statement (a), and statement (b) are obvious. It is clear that the image $N_{r}^{1}(u)$ under the natural homomorphism from $N$ onto $W$ is just $W_{r}^{1}$ for $r \in \Phi^{*}, u \in K_{r}^{*}$, so statement (c) follows.

It follows from the properties of the Satake diagrams that $\Gamma_{+}$is the subspace spanned by $\tilde{\Phi}^{\prime}$ over $\mathbb{R}$. It is easily verified that $W\left(\tilde{\Phi}^{\prime}\right)=W(\tilde{\Phi})^{\prime}=w\left(\Pi^{*}\right)^{\prime}$ where $W\left(\Pi^{*}\right)=\left\langle W_{r}^{1}, r \in \Pi^{*}\right\rangle$ and $W\left(\Pi^{*}\right)^{\prime}$ is the restriction of $W\left(\Pi^{*}\right)$ to $\Gamma_{+}$. Thus by Proposition 2(a) the statement (d) is established. The proof is complete.

\section{Corollary 1.}

(a) Let $r \in \Sigma_{1}^{*}, T, T_{1}, T_{2} \in K_{(r)}$ and $h \in H^{1}$. Then $X_{r}\left(T_{1}\right) X_{r}\left(T_{2}\right)=X_{r}(U)$, $U \in K_{(r)}, h X_{r}(T) h^{-1}=X_{r}\left(T^{\prime}\right), T^{\prime} \in K_{(r)}$.

(b) Let $r \in \Sigma_{1}^{*+} \cap I\left(r_{1}\right), r_{1} \in \Pi^{*}$ and $U, T \in K_{(r)}$. Let $u^{\prime}=N_{r}^{1} u\left(N_{r}^{1}\right)^{-1}$ and $u^{\prime \prime}=X_{-r}(U) u X_{-r}(-U)$, and $v^{\prime}=N_{r}^{1} v\left(N_{r}^{1}\right)^{-1}$ and $v^{\prime \prime}=X_{r}(T) v X_{r}(-T)$ where $u \in U^{1}$ and $v \in V^{1}$ satisfying $r \notin J(u)$ and $-r \notin J(v)$. Then $u^{\prime}, u^{\prime \prime} \in U^{1}$ and $v^{\prime}$, $v^{\prime \prime} \in V^{1}$ satisfying $r \notin J\left(u^{\prime}\right) \cup J\left(u^{\prime \prime}\right)$ and $-r \notin j\left(v^{\prime}\right) \cup J\left(v^{\prime \prime}\right)$ respectively.

Proof. Statement (a) is obvious.

(b) First we shall show that statements (1) and (2) below are true. Let $s \in \Sigma_{1}^{*+}$, $r \neq s$ and $T^{\prime}, U^{\prime} \in K_{(s)}$. Then

(1) $N_{r}^{1} X_{s}\left(U^{\prime}\right)\left(N_{r}^{1}\right)^{-1}=X_{s_{1}}\left(U_{1}\right), U_{1} \in K_{\left(s_{1}\right)}, s_{1} \neq r, s_{1} \in \Sigma_{1}^{*+}$,

(2) the element $u^{*}$ defined by $u^{*}=X_{-r}\left(T^{\prime}\right) X_{s}\left(U^{\prime}\right) X_{-r}(-T)$ belongs to $U^{1}$ and satisfies $r \notin J\left(u^{*}\right)$.

The proof of the statements (1) and (2) is given in the following.

(1) Since $r \in I\left(r_{1}\right), r_{1} \in \Pi^{*}$, we have $W_{r}^{1}(s) \in \Phi^{*+}$ and $W_{r}^{1}(s) \notin I(r)$. Thus we have $N_{r}^{1} X_{s}\left(U^{\prime}\right)\left(N_{r}^{1}\right)^{-1}=X_{s_{1}}\left(U_{1}\right)$ where $s_{1} \in I\left(W_{r}^{1}(s)\right) \cap \Sigma_{1}^{*+}, s_{1} \neq r$ and $U_{1} \in K_{\left(s_{1}\right)}$, so statement (1) follows.

(2) Let $s^{*} \in I(s)$ and $r^{*} \in I(-r)$. Then the roots is ${ }^{*}+j r^{*}, i, j$ being a pair of positive integers, must belong to $\Phi^{*+}$ since $r \in I\left(r_{1}\right), r_{1} \in \Pi^{*}$. Thus by Chevalley's commutator formula we have $u^{*} \in U$, so we have $u^{*} \in U^{1}$ since 
$\sigma u^{*}=u^{*}$. It is clear that $\left(i s^{*}+j r^{*}\right)^{\prime} \neq k r^{\prime}, k=1,2, \frac{1}{2}$. Hence we have $r \notin$ $J\left(u^{*}\right)$, so statement (2) follows.

By statements (1) and (2) we have $u^{\prime}, u^{\prime \prime} \in U^{1}$, satisfying $r \notin J\left(u^{\prime}\right) \cup J\left(u^{\prime \prime}\right)$.

Similarly, we have $v^{\prime}, v^{\prime \prime} \in V^{1}$, satisfying $-r \notin J\left(v^{\prime}\right) \cup J\left(v^{\prime \prime}\right)$.

We define $G_{0}=\left\langle x_{\alpha}(t), \alpha \in \Phi^{0}, t \in K ; H\right\rangle, Y^{1}=G^{1} \cap G_{0}$ and $B^{1}=Y^{1} U^{1}$.

\section{PROPOSITION 5.}

(a) Let $y \in Y^{1}, h \in H^{1}$ and $n \in N^{1}$. Then $\sigma y=y$ and $h y h^{-1}, n y n^{-1} \in Y^{1}$.

(b) $H^{1} \subset Y^{1} \subset G^{1}$.

(c) For each $r \in \Phi^{*}, h_{r}^{1}(v) \in H^{1}, v \in K_{r}^{* \prime}$.

(d) Let $r \in \Sigma_{1}^{*}, T \in K_{(r)}$ and $y \in Y^{1}$. Then $y X_{r}(T) y^{-1}=X_{r}\left(T^{\prime}\right), T^{\prime} \in K_{(r)}$.

(e) Let $u \in U^{1}, v \in V^{1}$ and $y \in Y^{1}$. Then $y u y^{-1} \in U^{1}$ and $y v y^{-1} \in V^{1}$.

Proof. If $w \in W^{1}$ and $\beta \in \Phi^{0}$ then we have $\rho_{\theta}^{\prime} w=w \rho_{\theta}^{\prime}$ and $\beta^{\prime}=0$, so we have $(w(\beta))^{\prime}=0$. It follows that $n y n^{-1} \in Y^{1}$. The other parts of statement (a), and statement (b) are clear. Statement (c) is obvious for all $r \in \Phi^{*} \backslash \Phi_{11 b}$. By 13.7.2 of [1] (cf. the case in which $J$ has type $A_{2}$ ) we have $h_{r}^{1}(v) \in H^{1}$ for all $r \in \Phi_{11 b}, v \in K_{r}^{* \prime}$ since $J=\{r, \bar{r}, r+\bar{r}\}$ has type $A_{2}$ for all $r \in \Phi_{11 b}$ also. We shall prove statement (d). Let $r^{*} \in I(r)$ and $\beta \in \Phi^{0}$. Then the roots $i r^{*}+j \beta$, $i, j$ being a pair of positive integers must belong to $I(r)$ since $\beta^{\prime}=0$, so statement (d) follows. By (d), statement (e) is established immediately.

Corollary 2. $B^{1}$ is a subgroup of $G^{1}$.

\section{The quasi-Bruhat decomposition of $L\left(\Pi^{\theta}, K\right)$}

For each $r \in \Pi^{*}$, there exists a maximal sub-diagram $\left(\Pi_{r}, \theta\right)$ of the Satake diagram $(\Pi, \theta)$ such that the restriction $\Pi_{r}^{\prime}$ of $\Pi_{r} \cap \Pi^{*}$ to $\Gamma_{+}$is a basis of the root system $I(r)^{\prime} \cup-I(r)^{\prime}$ (cf. pp. $456-458$ of [4]). A Satake diagram $(\Pi, \theta)$ is said to have $\mathbb{R}$-rank 1 if the dimension of $\Gamma_{+}$spanned by $\{r+\bar{r} \mid r \in \Pi\}$ over $\mathbf{R}$ is equivalent to 1 . Clearly, for each $r \in \Pi^{*}$, the Satake diagram $\left(\Pi_{r}, \theta\right)$ is a Satake diagram of $\boldsymbol{R}$-rank 1 . All Satake diagrams of $\mathbb{R}$-rank 1 are given in Table I. We shall consider separately the Satake diagrams of $\mathbb{R}$-rank 1 .

(1) $\Pi_{r} \cong \Pi\left(\mathbf{A}_{1}\right)\left(\right.$ cf. Table $\left.\mathrm{I}_{1}\right): I_{1}(r)=I(r)=\{r\}, r=\bar{r}$.

(2) $\Pi_{r}=\Pi\left(\mathbf{A}_{1} \times \mathbf{A}_{1}\right)\left(\mathrm{cf}\right.$. Table $\left.I_{2}\right): I_{1}(r)=\{r\}, I(r)=\{r, \bar{r}\}, r \neq \tilde{r}$.

(3) $\Pi_{r}=\Pi\left(\mathbf{A}_{3}\right)$ (cf. Table $\left.I_{3}\right): I_{1}(r)=\left\{r=e_{2}-e_{3}=r_{1}, r^{*}=e_{2}-e_{4}=r_{2}\right\}$, $I(r)=\left\{r_{1}, r_{2}, \bar{r}_{1}=e_{1}-e_{4}, \bar{r}_{2}=e_{1}-e_{3}\right\}$. Let $\alpha_{1}=e_{1}-e_{2}$ and $\alpha_{2}=e_{3}-e_{4}$. 
We have $-\bar{r}_{2}+r_{1}+\alpha_{1}=0$ and $r_{1}-r_{2}+\alpha_{2}=0$, so by 4.1 .2 of [1] we have

$$
\begin{aligned}
& N_{\alpha_{1},-\bar{r}_{2}}=N_{-\bar{r}_{2}, r_{1}}=N_{r_{1}, \alpha_{1}}=\xi_{1}, \quad N_{r_{1},-r_{2}}=N_{-r_{2}, \alpha_{2}}=N_{\alpha_{2}, r_{1}}=\xi_{2}, \\
& N_{-\alpha_{1}, r_{2}}=N_{-r_{2}, \bar{r}_{1}}=N_{\bar{r}_{1},-\alpha_{1}}=\xi_{3}, \quad N_{\bar{r}_{1},-\bar{r}_{2}}=N_{-\bar{r}_{1},-\alpha_{2}}=N_{-\alpha_{2}, \bar{r}_{1}}=\xi_{4} .
\end{aligned}
$$

By (B), we have $N_{\bar{r}_{2}, \alpha_{2}} k_{r_{2}} k_{-\alpha_{2}}=N_{r_{2},-\alpha_{2}} k_{\bar{r}_{1}}$. It follows that $-\xi_{2} \xi_{4}=1$ since $k_{\bar{r}_{1}}=k_{r_{2}}=1$ and $k_{-\alpha_{2}}=-1$. Similarly, we have $-\xi_{1} \xi_{3}=1$.

We shall denote by $I_{k}$ the set $\{1,2, \ldots, k-1\}$ and by $m$ an integer satisfying $1 \leqslant m \leqslant \mathbf{n}, \mathbf{n}$ being the rank of $\Pi_{r}, r \in \Pi^{*}$.

(4) $\Pi_{r}=\Pi\left(\mathbf{A}_{\mathrm{n}}\right)$ (cf. Table $\left.\mathrm{I}_{4}\right): r=e_{1}-e_{2}, s=e_{\mathrm{n}}-e_{\mathrm{n}+1}, r_{0}=e_{1}-e_{\mathrm{n}+1}=$ $r_{\mathrm{n}}=r+\bar{r} . I_{1}(r)=\left\{r_{0}, r_{i}=e_{i+1}-e_{\mathrm{n}+1}, i \in I_{\mathrm{n}}\right\}, I(r)=\left\{r_{0}, r_{i}, \bar{r}_{i}=e_{1}+e_{i+1}, i\right.$ $\left.\in I_{\mathrm{n}}\right\}$. Let $\beta_{i}=e_{i+1}-e_{m+1}, i \in I_{m}$. Then we have $\beta_{i}-r_{i}+r_{m}=0$ and $-\beta_{i}-\bar{r}_{i}$ $+\bar{r}_{m}=0, i \in I_{m}$, so by 4.1 .2 of [1] we have

$N_{\beta_{i},-r_{i}}=N_{-r_{i}, r_{m}}=N_{r_{m}, \beta_{i}}=\xi_{i}^{\prime}, \quad N_{-\beta_{i},-\tilde{r}_{i}}=N_{-\tilde{r}_{i}, \bar{r}_{m}}=N_{\bar{r}_{m},-\beta_{i}}=-\xi_{i}, \quad i \in I_{m}$.

By (B) we have $\xi_{i} \xi_{i}^{\prime}=1$. Since $-r_{0}+r_{i}+\bar{r}_{i}=0$ we have $N_{-r_{0}, r_{i}}=N_{r_{i}, \bar{r}_{i}}=N_{\bar{r}_{i},-r_{0}}$ $=\eta, i \in I_{n}$. It is clear that $r_{i}+\bar{r}_{i}-r_{m}-\bar{r}_{m}=0, i \in I_{m}$. Thus by 4.1.2 of [1] we have $N_{r_{i}, \bar{r}_{i}} N_{-r_{m},-\bar{r}_{m}}=-N_{-r_{m}, r_{i}} N_{\bar{r}_{i},-\bar{r}_{m}} i \in I_{m}$. It follows from (B) that $-N_{-r_{m}, r_{i}}=$ $N_{-\bar{r}_{m}, \bar{r}_{i}}, i \in I_{m}$, so we have $N_{r_{i}, \bar{r}_{i}}=N_{r_{m}, \bar{r}_{m}}=\eta, i \in I_{m}$.

(5)-(6). $\Pi_{r} \cong \Pi\left(\mathbf{B}_{\mathbf{n}}\right)$ or $\Pi\left(\mathbf{D}_{\mathbf{n}}\right)$ (cf. Table $\left.\mathbf{I}_{5}, \mathbf{I}_{6}\right): r=e_{1}-e_{2}$. We write $r_{i}=e_{1}-e_{i+1}$ and $\bar{r}_{i}=e_{1}+e_{i+1}, i \in I_{\mathrm{n}}$ and $\alpha_{j}=e_{j+1}+e_{m+1}$ and $\beta_{j}=-e_{j+1}$ $+e_{m+1}, j \in I_{m}$. We have $r_{\mathrm{n}+1}=e_{1}$. Let $r_{0}$ denote $e_{1}$ and $\alpha_{0}$ denote $-e_{m+1}$.

$\Pi_{r}=\Pi\left(\mathbf{B}_{\mathrm{n}}\right): I_{1}(r)=\left\{r_{0}, r_{i}, i \in I_{\mathrm{n}}\right\}, I(r)=\left\{r_{0}, r_{i}, \bar{r}_{i}, i \in I_{n}\right\}$.

$\Pi_{r}=\Pi\left(\mathbf{D}_{\mathrm{n}}\right): I_{1}(r)=\left\{r_{i}, i \in I_{\mathrm{n}}\right\}, I(r)=\left\{r_{i}, \bar{r}_{i}, I \in I_{n}\right\}$. We have $\alpha_{i}+r_{m}-$ $\bar{r}_{i}=0$ and $\beta_{i}+r_{m}-r_{i}=0, i \in I_{m}$. By 4.1.2 of [1] and (B) we have

$$
\begin{array}{ll}
N_{\alpha_{i},-\bar{r}_{i}}=N_{-\bar{r}_{i}}, r_{m}=N_{r_{m}, \alpha_{i}}=\xi_{i}^{\prime}, & N_{-\alpha_{i},-r_{i}}=N_{-r_{i}, \bar{r}_{m}}=N_{\bar{r}_{m},-\alpha_{i}}=-\xi_{i}, \\
N_{\beta_{i},-r_{i}}=N_{-r_{i}, r_{m}}=N_{r_{m}, \beta_{i}}=\eta_{i}^{\prime}, & N_{-\beta_{i},-\bar{r}_{i}}=N_{-\bar{r}_{i}, \bar{r}_{m}}=N_{\bar{r}_{m},-\beta_{i}}=-\eta_{i} .
\end{array}
$$

Moreover, we have $\xi_{i} \xi_{i}^{\prime}=1$ and $\eta_{i} \eta_{i}^{\prime}=1$ for $i \in I_{m}$. It is clear that $r_{m}-r_{0}-\alpha_{0}$ $=0$ and $\bar{r}_{m}-r_{0}+\alpha_{0}=0$, so by 4.1 .2 of [1] we have $N_{r_{m},-r_{0}}=N_{-\alpha_{0}, r_{m}}=\frac{1}{2} N_{-r_{0},-\alpha_{0}}$ $=\eta_{0}, N_{r_{m},-r_{0}}=N_{\alpha_{0}, \bar{r}_{m}}=\frac{1}{2} N_{-r_{0}, \alpha_{0}}=\eta_{0}$, and $\eta_{0} \eta_{0}^{\prime}= \pm 1$.

Clearly, all $\xi_{i}, \xi_{i}^{\prime}, \eta_{i}, \eta_{i}^{\prime}, \eta$ and $\eta^{\prime}$ mentioned in 1-6 are equal to \pm 1 .

We obtain statements (2A) and (2B) immediately.

(2A) For each $r \in \Pi^{*}, s \in I(r)$, we have $(s, \bar{s})=0$ if $\Pi_{r} \not \equiv \Pi\left(\mathbf{A}_{\mathbf{n}}\right)$, and we have $A_{s \bar{s}}=A_{\bar{s} s}=-1$ and $s \neq \bar{s}$ if $\Pi_{r} \cong \Pi\left(\mathrm{A}_{\mathrm{n}}\right)$ (cf. Table $\mathrm{I}_{4}$ ).

(2B) For each $r \in \Pi^{*}, I(r)=\left\{s \in \Phi^{*+} \mid W_{r}^{1}(s) \in \phi^{-}\right\}$.

Lemma 1. Let $r \in \Sigma_{1}^{*+} \cap I\left(r_{1}^{*}\right), r_{1}^{*} \in \Pi^{*}, I_{1}(r)=\left\{r_{1}, r_{2}, \ldots, r_{n}\right\}$ and $T=$ $\left(t_{1}, t_{2}, \ldots, t_{n}\right) \in K_{(r)}$. If $D(T) \neq 0$ then $X_{-r}(T)=X_{r}\left(T^{*}\right) N_{r}^{1} X_{r}\left(T^{* \prime}\right) y=$ $y^{\prime} X_{r}\left(T^{\prime}\right) N_{r}^{1} X_{r}\left(T^{\prime \prime}\right), T^{*}, T^{* \prime}, T^{\prime}, T^{\prime \prime} \in K_{(r)}, y^{\prime}, y \in Y^{1}$. 
Proof. We shall consider the cases $1-6$ separately.

1. Table $\mathrm{I}_{1}: X_{-r}(T)=X_{-r}^{1}(t)=x_{-_{r}}(t), T=(t) \in K_{(r)}$. Since $D(T) \neq 0$ we have $t \neq 0$, so we have

$$
\begin{aligned}
X_{-r}(T) & =x_{r}\left(t^{-1}\right) h_{r}\left(-t^{-1}\right) n_{r} x_{r}\left(t^{-1}\right) \\
& =X_{r}\left(T^{*}\right) N_{r}^{1} X_{r}\left(T^{* \prime}\right) y,
\end{aligned}
$$

(by 6.4 of [1])

where $y=h_{r}(-t)=h_{r}^{1}(-t) \in H^{1} \subset Y^{1}$ and $n_{r}=N_{r}^{1} \in N^{1}$, and $T^{*}=\left(t^{-1}\right), T^{* \prime}$ $=(t) \in K_{(r)}$.

2. Table $\mathrm{I}_{2}: X_{-r}(T)=X_{-r}^{1}(t)=x_{-r}(t) x_{-\bar{r}}(\bar{t}), T=(t) \in K_{(r)}$. Since $D(T) \neq 0$ we have $t \neq 0$, so we have

$$
X_{-r}(T)=x_{r}\left(t^{-1}\right) x_{\tilde{r}}\left(\bar{t}^{-1}\right) h_{r}\left(-t^{-1}\right) h_{\bar{r}}\left(-\bar{t}^{-1}\right) n_{r} n_{\bar{r}} x_{r}\left(t^{-1}\right) x_{\bar{r}}\left(\bar{t}^{-1}\right)
$$

(by 6.4 .4 of $[1]$ )

$$
=X_{r}\left(T^{*}\right) N_{r}^{1} X_{r}\left(T^{* \prime}\right) y
$$

where $y=h_{r}(-t) h_{r}(\bar{t}) \in Y^{1}$ and $n_{r} n_{\bar{r}}=N_{r}^{1} \in N^{1}$, and $T^{*}=\left(t^{-1}\right), T^{* \prime}=(t)$ $\in K_{(r)}$.

3. Table $I_{3}: X_{-r}(T)=X_{-r_{1}}^{1}\left(t_{1}\right) X_{-r_{2}}^{1}\left(t_{2}\right)=x_{-r_{1}}\left(t_{1}\right) x_{-\bar{r}_{1}}\left(\bar{t}_{1}\right) x_{-r_{2}}\left(t_{2}\right) x_{-\bar{r}_{2}}\left(\bar{t}_{2}\right), T=$ $\left(t_{1}, t_{2}\right) \in K_{(r)}$. Since $D(T) \neq 0$ we have $T \neq(0,0)$. If either $t_{1}$ or $t_{2}$ is equal to zero the lemma is true as in the case of Table $I_{2}$. We assume that $t_{1} \neq 0$ and $t_{2} \neq 0$. Then by 6.4 .4 and 5.2 .2 of [1] we have

$$
\begin{aligned}
& X_{-r}(T)=x_{\bar{r}_{1}}\left(\bar{t}_{1}^{-1}\right) h_{\bar{r}_{1}}\left(-\bar{t}_{1}^{-1}\right) n_{\bar{r}_{1}}\left(\bar{t}_{1}^{-1}\right) x_{\bar{r}_{1}}\left(\bar{t}_{1}^{-1}\right) x_{-r_{1}}\left(t_{1}\right) x_{-r_{2}}\left(t_{2}\right) x_{-\bar{r}_{2}}\left(\bar{t}_{2}\right) \\
& =x_{\bar{r}_{1}}\left(\bar{t}_{1}^{-1}\right) h_{\bar{r}_{1}}\left(-\bar{t}_{1}^{-1}\right) n_{\bar{r}_{1}} x_{-r_{1}}\left(t_{1}\right) x_{-r_{2}}\left(t_{2}\right) x_{\alpha_{1}}\left(-\xi_{3} \bar{t}_{1}^{-1} t_{2}\right) x_{-\bar{r}_{2}}\left(\bar{t}_{2}\right) x_{\alpha_{2}}\left(\xi_{4} \bar{t}_{1}^{-1} \bar{t}_{2}\right) x_{\bar{r}_{1}}\left(\bar{t}_{1}^{-1}\right)
\end{aligned}
$$

(by 5.2 .2 of [1])

$$
=x_{\bar{r}_{1}}\left(\bar{t}_{1}^{-1}\right) h_{\bar{r}_{1}}\left(-\bar{t}_{1}^{-1}\right) n_{\bar{r}_{1}} x_{-r_{1}}(d) x_{-\bar{r}_{2}}\left(\bar{t}_{2}\right) x_{r_{1}}\left(\bar{t}_{1}^{-1}\right) y_{1}
$$

where $d=t_{1}-\xi_{1} \xi_{3} \bar{t}_{1}^{-1} t_{2} \bar{t}_{2}$ and $y_{1}=x_{\alpha_{1}}\left(-\xi_{3} \tilde{t}_{1}^{-1} t_{2}\right) x_{\alpha_{2}}\left(\xi_{4} \tilde{t}_{1}^{-1} \bar{t}_{2}\right)$. Since $D(T) \neq 0$ and $-\xi_{1} \xi_{3}=1$ we have $d=\bar{t}_{1}^{-1}\left(t_{1} \bar{t}_{1}+t_{2} \bar{t}_{2}\right)=\bar{t}_{1}^{-1} D(T) \neq 0$. Thus we have

$$
\begin{aligned}
& X_{-r}(T)=x_{\bar{r}_{1}}\left(\bar{t}_{1}^{-1}\right) x_{r_{1}}\left(d^{-1}\right) h_{r_{1}}\left(-d^{-1}\right) n_{\bar{r}_{1}} n_{r_{1}} x_{-r_{2}}\left(t_{2}\right) x_{-\alpha_{2}}\left(\xi_{2} d^{-1} t_{2}\right), \\
& x_{-\bar{r}_{2}}\left(\bar{t}_{2}\right) x_{-\alpha_{1}}\left(-\xi_{1} d^{-1} \bar{t}_{2}\right) x_{r_{1}}\left(d^{-1}\right) x_{\bar{r}_{1}}\left(\bar{t}_{1}^{-1}\right) y_{1} \\
& \quad=x_{\bar{r}_{1}}\left(\bar{t}_{1}^{-1}\right) x_{r_{1}}\left(d^{-1}\right) h_{r_{1}}\left(-\bar{t}_{1}^{-1}\right) h_{r_{1}}\left(-d^{-1}\right) x_{\bar{r}_{2}}\left(t_{2}\right) x_{r_{2}}\left(\bar{t}_{2}\right) x_{\bar{r}_{1}}\left(-d^{-1} t_{2} \bar{t}_{2}\right), \\
& n_{\bar{r}_{1}} n_{r_{1}} x_{r_{1}}\left(d^{-1}\right) x_{\bar{r}_{1}}\left(\bar{t}_{1}^{-1}\right) x_{r_{1}}\left(\xi_{2}^{2} d^{-2} \bar{t}_{1}^{-1} t_{2} \bar{t}_{2}\right) x_{\bar{r}_{2}}\left(-d^{-1} \bar{t}_{1}^{-1} t_{2}\right) x_{r_{2}}\left(-d^{-1} \bar{t}_{1}^{-1} \bar{t}_{2}\right) y_{2} y_{1}
\end{aligned}
$$

where $y_{2}=x_{-\alpha_{1}}\left(\xi_{3} d^{-1} \bar{t}_{2}\right) x_{-\alpha_{2}}\left(-\xi_{4} d^{-1} t_{2}\right)$. Finally we have

$$
\begin{aligned}
X_{-r}(T) & =x_{r_{1}}\left(t_{1}^{*}\right) x_{\bar{r}_{1}}\left(\bar{t}_{1}^{*}\right) x_{r_{2}}\left(t_{2}^{*}\right) x_{\bar{r}_{2}}\left(\bar{t}_{2}^{*}\right) n_{\bar{r}_{1}} n_{r_{1}} x_{r_{1}}\left(t_{1}^{* \prime}\right) x_{\bar{r}_{1}}\left(\bar{t}_{1}^{* \prime}\right) x_{r_{2}}\left(t_{2}^{* \prime}\right) x_{\bar{r}_{2}}\left(\bar{t}_{2}^{* \prime}\right) y \\
& =X_{r_{1}}^{1}\left(t_{1}^{*}\right) X_{r_{2}}^{1}\left(t_{2}^{*}\right) N_{r_{1}}^{1} X_{r_{1}}^{1}\left(t_{1}^{* \prime}\right) X_{r_{2}}^{1}\left(t_{2}^{* \prime}\right) y=X_{r}\left(T^{*}\right) N_{r}^{1} X_{r}\left(T^{* \prime}\right) y
\end{aligned}
$$

where $y=h_{\bar{r}_{1}}\left(-\bar{t}_{1}\right) h_{r_{1}}(-d) y_{2} y_{1}$ and $n_{r_{1}} n_{\bar{r}_{1}}=N_{r}^{1} \in N^{1} \quad\left(r_{1}=r\right)$, and $t_{1}^{*}=$ $\bar{t}_{1} D(T)^{-1}, t_{2}^{*}=\bar{t}_{2} D(T)^{-1}, t_{1}^{* \prime}=t_{1}, t_{2}^{* \prime}=t_{2} \in K_{r}$ and $T^{*}=\left(t_{1}^{*}, t_{2}^{*}\right), T^{* \prime}=$ $\left(t_{1}^{* \prime}, t_{2}^{* \prime}\right) \in K_{(r)}$. Clearly, we have $y \in G_{0}$. Since $X_{-r}(T), X_{r}\left(T^{*}\right), X_{r}\left(T^{* \prime}\right)$, $N_{r}^{1} \in G^{1}$, so $y \in Y^{1}$. 
4. Table $\mathrm{I}_{4}: X_{-r}(T)=X_{-r_{0}}^{1}\left(t_{0}\right) X_{-r_{1}}^{1}\left(t_{1}\right) \cdots X_{-r_{n}}^{1}\left(t_{n}\right)$ where $t_{0}=-\frac{1}{2} \eta \tilde{D}(T)-\varepsilon$, $\tilde{D}(T)=t_{1} \bar{t}_{1}+t_{2} \bar{t}_{2}+\cdots+t_{n-1} \bar{t}_{n-1}, \varepsilon \in K_{r_{0}}=\{t \in K \mid t=-\bar{t}\},\left(r_{n}=r_{0}\right)$. Since $D(T) \neq 0$ we have $T \neq(0,0, \ldots, 0)$, so there exists an integer $m$ satisfying $1 \leqslant m \leqslant n$ such that $t_{n-1}=t_{n-2}=\cdots=t_{m+1}=0$ and $t_{m} \neq 0$ (we write $t_{m}=$ t). By 6.4.4 and 5.2.2 of [1] we have

$$
\begin{array}{r}
X_{-r}(T)=x_{-\bar{r}_{m}}(\bar{t}) x_{-r_{m}}(t) x_{-r_{0}}\left(t_{0}\right) x_{-\bar{r}_{1}}\left(\bar{t}_{1}\right) x_{-r_{1}}\left(t_{1}\right) \cdots x_{-\bar{r}_{m-1}}\left(\bar{t}_{m-1}\right) x_{-r_{m-1}}\left(t_{m-1}\right) \\
=x_{\dot{r}_{m}}\left(\bar{t}^{-1}\right) h_{\bar{r}_{m}}\left(-\bar{t}^{-1}\right) n_{\bar{r}_{m}} x_{-r_{m}}(t) x_{-r_{0}}\left(t_{0}\right) x_{-r_{m}}\left(\eta \bar{t}^{-1} t_{0}\right) x_{-\bar{r}_{1}}\left(\bar{t}_{1}\right) x_{\beta_{1}}\left(\xi_{1} \bar{t}^{-1} \bar{t}_{1}\right), \\
x_{-r_{1}}\left(t_{1}\right) \cdots x_{-\bar{r}_{m-1}}\left(\bar{t}_{m-1}\right) x_{\beta_{m-1}}\left(\xi_{m-1} \bar{t}^{-1} \bar{t}_{m-1}\right) x_{-r_{m-1}}\left(t_{m-1}\right) x_{\bar{r}_{m}}\left(\bar{t}^{-1}\right) \\
=x_{\bar{r}_{m}}\left(\bar{t}^{-1}\right) h_{\bar{r}_{m}}\left(-\bar{t}^{-1}\right) n_{\bar{r}_{m}} x_{-r_{m}}\left(\bar{t}^{-1} t_{0}^{*}\right) x_{-r_{0}}\left(t_{0}\right) x_{-\bar{r}_{1}}\left(\bar{t}_{1}\right) x_{-r_{1}}\left(t_{1}\right) \cdots \\
x_{-\bar{r}_{m-1}}\left(\bar{t}_{m-1}\right) x_{-r_{m-1}}\left(t_{m-1}\right) x_{\bar{r}_{m}}\left(\bar{t}^{-1}\right) y_{1}
\end{array}
$$

where

$$
y_{1}=x_{\beta_{1}}\left(\xi_{1} \bar{t}^{-1} \bar{t}_{1}\right) \cdots x_{\beta_{m-1}}\left(\xi_{m-1} \bar{t}^{-1} \bar{t}_{m-1}\right)
$$

and

$$
t_{0}^{*}=\bar{t}^{-1}\left(\frac{1}{2} \tilde{D}(T)+\eta \varepsilon\right)=\bar{t}^{-1} \eta \bar{t}_{0} .
$$

We write $u=-\eta \hat{t}^{-1} \tilde{t}_{0}$ and $v=-\eta u^{-1} t_{0}$. Since $D(T) \neq 0$ we have $t_{0} \neq 0$ and $t_{0}^{*} \neq 0$, so we have $u \neq 0$ and $v \neq 0$. Thus we have

$$
\begin{aligned}
X_{-r}(T)= & x_{\bar{r}_{m}}\left(\bar{t}^{-1}\right) h_{\dot{r}_{m}}\left(\bar{t}^{-1}\right) x_{r_{0}}\left(u^{-1}\right) h_{r_{0}}\left(-u^{-1}\right) n_{\bar{r}_{m}} n_{r_{m}} x_{-r_{0}}\left(t_{0}\right) x_{-\bar{r}_{m}}(v) x_{-\bar{r}_{1}}\left(\bar{t}_{1}\right) \\
& \times x_{-r_{1}}\left(t_{1}\right) x_{-\beta_{1}}\left(-\xi_{1}^{\prime} u^{-1} t_{1}\right) \cdots x_{-\bar{r}_{m-1}}\left(\bar{t}_{m-1}\right) x_{-r_{m-1}}\left(t_{m-1}\right) \\
& \times x_{-\beta_{m-1}}\left(-\xi_{m-1}^{*} u^{-1} t_{m-1}\right) x_{r_{m}}\left(u^{-1}\right) x_{\bar{r}_{m}}\left(\bar{t}^{-1}\right) y_{1} \\
= & x_{\bar{r}_{m}}\left(\bar{t}^{-1}\right) h_{\bar{r}_{m}}\left(-\bar{t}^{-1}\right) x_{r_{0}}\left(u^{-1}\right) h_{r_{0}}\left(-u^{-1}\right) x_{r_{m}}\left(v^{-1}\right) h_{r_{m}}\left(-v^{-1}\right) n_{\bar{r}_{m}} n_{r_{m}} n_{\bar{r}_{m}} x_{-r_{m}}\left(\tilde{t}^{*}\right) \\
& \times x_{-r_{0}}\left(t_{0}\right) x_{-\bar{r}_{1}}\left(\bar{t}_{1}\right) x_{-r_{1}}\left(t_{1}\right) \cdots x_{-\bar{r}_{m-1}}\left(t_{m-1}\right) x_{-r_{m-1}}\left(t_{m-1}\right) \\
& \times x_{\bar{r}_{m}}\left(v^{-1}\right) y_{3} x_{r_{m}}\left(u^{-1}\right) y_{2} x_{\bar{r}_{m}}\left(\bar{t}^{-1}\right) y_{1} \\
= & x_{\bar{r}_{m}}\left(\bar{t}^{-1}\right) h_{\bar{r}_{m}}\left(-\bar{t}^{-1}\right) x_{r_{0}}\left(u^{-1}\right) h_{r_{0}}\left(-u^{-1}\right) x_{r_{m}}\left(v^{-1}\right) h_{r_{m}}\left(-v^{-1}\right) \\
& \times x_{\bar{r}_{m}}\left(\tilde{t}^{*}\right) x_{r_{0}}\left(t_{0}\right) x_{r_{1}}\left(\bar{t}_{1}\right) x_{\bar{r}_{1}}\left(t_{1}\right) \cdots x_{r_{m-1}}\left(\bar{t}_{m-1}\right) x_{\bar{r}_{m-1}}\left(t_{m-1}\right) \\
& \times n_{\bar{r}_{m}} n_{r_{m}} n_{\bar{r}_{m}} x_{\bar{r}_{m}}\left(v^{-1}\right) y_{3} x_{r_{m}}\left(u^{-1}\right) y_{2} x_{\bar{r}_{m}}\left(\bar{t}^{-1}\right) y_{1} .
\end{aligned}
$$

where $\tilde{t}^{*}=v^{-1}\left(\eta(\tilde{D}(T)-t \bar{t})+t_{0}\right)$, and $y_{2}=x_{-\beta_{1}}\left(-\xi_{1}^{\prime} u^{-1} t_{1}\right) \cdots$ $x_{-\beta_{m-1}}\left(-\xi_{m-1}^{\prime} u^{-1} t_{m-1}\right)$ and $y_{3}=x_{\beta_{1}}\left(\xi_{1} v^{-1} \bar{t}_{1}\right) \cdots x_{\beta_{m-1}}\left(\xi_{m-1} v^{-1} \bar{t}_{m-1}\right)$. Finally, we have $X_{-r}(T)=X_{r}\left(T^{*}\right) N_{r}^{1} X_{r}\left(T^{* \prime}\right) y$ where $y=\bar{h}^{-1} h_{r_{m}}(-\bar{t}) h_{r_{0}}(-u) h_{\bar{r}_{m}}(-v) y_{3} y_{2} y_{1}$ $\in Y^{1}$ and $N_{r}^{1}=\bar{h} n_{r_{m}} n_{r_{m}} n_{r_{m}}=\bar{h} n_{r_{m}+\bar{r}_{m}}=\bar{h} n_{r} \in N^{1}, \bar{h} \stackrel{{ }^{m}}{\in} H^{1}$ and $T^{*}, T^{* \prime} \in K_{(r)}$.

5. Table $\mathrm{I}_{s}: X_{-r}(T)=X_{-r_{1}}^{1}\left(t_{1}\right) X_{-r_{2}}^{1}\left(t_{2}\right) \cdots X_{-r_{n}}^{1}\left(t_{n}\right)$. Since $D(T) \neq 0$ there exists an integer $m$ satisfying $1 \leqslant m \leqslant n$ such that $t_{n}=t_{n-1}=\cdots=t_{m+1}=0$ 
and $t_{m} \neq 0$. We shall write $t_{m}=t$. By 6.4.4 and 5.2.2 of [1] we have

$$
\begin{gathered}
X_{-r}(T)=x_{-\bar{r}_{m}}(\bar{t}) x_{-r_{m}}(t) x_{-\bar{r}_{1}}\left(\bar{t}_{1}\right) x_{-r_{1}}\left(t_{1}\right) \cdots x_{-\bar{r}_{m-1}}\left(\bar{t}_{m-1}\right) x_{-r_{m-1}}\left(t_{m-1}\right) \\
=x_{\bar{r}_{m}}\left(\bar{t}^{-1}\right) h_{\bar{r}_{m}}\left(-\bar{t}^{-1}\right) n_{\bar{r}_{m}} x_{-r_{m}}(t) x_{-r_{1}}\left(t_{1}\right) x_{\alpha_{i}}\left(\xi_{1} \bar{t}^{-1} t_{1}\right) x_{-\bar{r}_{1}}\left(\bar{t}_{1}\right) x_{\beta_{1}}\left(\eta_{1} \bar{t}^{-1} \bar{t}_{1}\right) \cdots \\
x_{-r_{m-1}}\left(t_{m-1}\right) x_{\alpha_{m-1}}\left(\xi_{m-1} \bar{t}^{-1} t_{m-1}\right) \\
\quad \times x_{-\bar{r}_{m-1}}\left(\bar{t}_{m-1}\right) x_{\beta_{m-1}}\left(\eta_{m-1} \bar{t}^{-1} \bar{t}_{m-1}\right) x_{r_{m}}\left(\bar{t}^{-1}\right) \\
=x_{\bar{r}_{m}}\left(\bar{t}^{-1}\right) h_{\bar{r}_{m}}\left(-\bar{t}^{-1}\right) n_{\bar{r}_{m}}(t) x_{-r_{m}}(t) x_{-r_{1}}\left(t_{1}\right) x_{-\bar{r}_{1}}\left(\bar{t}_{1}\right) x_{-r_{m}}\left(\xi_{1} \xi_{1}^{\prime} \bar{t}^{-1} t_{1} \bar{t}\right) \cdots \\
\quad x_{-r_{m-1}}\left(t_{m-1}\right) x_{-\bar{r}_{m-1}}\left(\bar{t}_{m-1}\right) x_{-r_{m}}\left(\xi_{m-1} \xi_{m-1}^{\prime} \bar{t}^{-1} t_{m-1} \bar{t}_{m-1}\right) x_{\bar{r}_{m}}\left(\bar{t}^{-1}\right) y_{1} \\
=x_{\bar{r}_{m}}\left(\bar{t}^{-1}\right) h_{\bar{r}_{m}}\left(-\bar{t}^{-1}\right) n_{\bar{r}_{m}} x_{-r_{m}}(d) x_{-r_{1}}\left(t_{1}\right) x_{-\bar{r}_{1}}\left(\bar{t}_{1}\right) \cdots \\
x_{-r_{m-1}}\left(t_{m-1}\right) x_{-\bar{r}_{m-1}}\left(\bar{t}^{-1}\right) x_{\bar{r}_{m}}\left(\bar{t}^{-1}\right) y_{1}
\end{gathered}
$$

where $y_{1}=x_{\alpha_{1}}\left(\xi_{1} \bar{t}^{-1} t_{1}\right) x_{\beta_{1}}\left(\eta_{1} \bar{t}^{-1} \bar{t}_{1}\right) \cdots x_{\alpha_{m-1}}\left(\xi_{m-1} \bar{t}^{-1} t_{m-1}\right) x_{\beta_{m-1}}\left(\eta_{m-1} \bar{t}^{-1} \bar{t}_{m-1}\right)$ and $d=\bar{t}^{-1} D(T)$. Since $D(T) \neq 0$ we have $d \neq 0$. By 6.4.4 and 5.2.2 of [1] we have

$$
\begin{aligned}
X_{-r}(T) & =x_{\bar{r}_{m}}\left(\bar{t}^{-1}\right) h_{\bar{r}_{m}}\left(-\bar{t}^{-1}\right) x_{r_{m}}\left(d^{-1}\right) h_{r_{m}}\left(-d^{-1}\right) n_{\bar{r}_{m}} n_{r_{m}} x_{-r_{1}}\left(t_{1}\right) x_{-\beta_{1}}\left(-r_{1}^{\prime} d^{-1} t_{1}\right) \\
& \times x_{-\bar{r}_{1}}\left(\bar{t}_{1}\right) x_{-\alpha_{1}}\left(-\xi_{1}^{\prime} d^{-1} \bar{t}_{1}\right) \cdots x_{-r_{m-1}}\left(t_{m-1}\right) x_{-\beta_{m-1}}\left(-\eta_{m-1}^{\prime} d^{-1} t_{m-1}\right) \\
& \times x_{-\bar{r}_{m-1}}\left(\bar{t}_{m-1}\right) x_{-\alpha_{m-1}}\left(-\xi_{m-1}^{\prime} d^{-1} \bar{t}_{m-1}\right) x_{r_{m}}\left(d^{-1}\right) x_{\bar{r}_{m}}\left(\bar{t}^{-1}\right) y_{1} \\
= & x_{\bar{r}_{m}}\left(\bar{t}^{-1}\right) h_{\bar{r}_{m}}\left(-\bar{t}^{-1}\right) x_{r_{m}}\left(d^{-1}\right) h_{r_{m}}\left(-d^{-1}\right) x_{\bar{r}_{m}}\left(d^{-1} t^{*}\right) x_{\bar{r}_{1}}\left(t_{1}\right) x_{r_{1}}\left(\bar{t}_{1}\right) \cdots \\
& x_{\bar{r}_{m-1}}\left(t_{m-1}\right) x_{r_{m-1}}\left(\bar{t}_{m-1}\right) n_{\bar{r}_{m}} n_{r_{m}} x_{r_{m}}\left(d^{-1}\right) y_{2} x_{\bar{r}_{m}}\left(\bar{t}^{-1}\right) y_{1}
\end{aligned}
$$

where

$$
\begin{aligned}
& y_{2}=x_{-\beta_{1}}\left(-\eta_{1}^{\prime} d^{-1} t_{1}\right) x_{-\alpha_{1}}\left(-\xi_{1}^{\prime} d^{-1} \bar{t}_{1}\right) \cdots \\
& x_{-\beta_{m-1}}\left(-\eta_{m-1}^{\prime} d^{-1} t_{m-1}\right) x_{-\alpha_{m-1}}\left(-\xi_{m-1}^{\prime} d^{-1} \tilde{t}_{m-1}\right)
\end{aligned}
$$

and

$$
t^{*}=D(T)-t t .
$$

Clearly, we have $N_{r}^{1}=n_{\bar{r}} n_{r}=n_{\bar{r}_{m}} n_{r_{m}} n_{\beta_{1}} n_{\alpha_{1}}$ or $N_{r}^{1}=n_{\bar{r}_{m}} n_{r_{m}}$ according as $r_{m} \neq r$ or $r_{m}=r$. We define $y^{*}=h_{\bar{r}_{m}}(-\bar{t}) h_{r_{m}}(-d) y_{2} y_{1}$. Furthermore, we write $y=$ $n_{\beta_{1}}^{-1} n_{\alpha_{1}}^{-1} y^{*}$ or $y=y^{*}$ according as $r_{m} \neq r$ or $r_{m}=r$. Finally, we have $X_{-r}(T)=$ $X_{r}\left(T^{*}\right) N_{r}^{1} X_{r}\left(T^{* \prime}\right) y$ where $y \in Y^{1}$ and $N_{r}^{1}=n_{\bar{r}} n_{r} \in N^{1}$, and $T^{*}, T^{* \prime} \in K_{(r)}$.

Table $\mathrm{I}_{6}$ : by a similar argument to that in the cases 4 and 5 , the following result can be proved to be true for this case: $X_{-r}(T)=X_{r}\left(T^{*}\right) N_{r}^{1} X_{r}\left(T^{* \prime}\right) y$ where $y \in Y^{1}, N_{r}^{1}=n_{r} n_{r} \in N^{1}$ and $T^{*}, T^{* \prime} \in K_{(r)}$. 
We may summarize the results of the cases 1-6 in the form $X_{-r}(T)=$ $X_{r}\left(T^{*}\right) N_{r}^{1} X_{r}\left(T^{* \prime}\right) y$ where $y \in Y^{1}$ and $T^{*}, T^{* \prime} \in K_{(r)}$.

Thus it follows from Proposition 5 that

$$
X_{-r}(T)=y^{\prime} X_{r}\left(T^{\prime}\right) N_{r} X_{r}\left(T^{\prime \prime}\right), \quad y^{\prime} \in Y^{1}, T^{\prime}, T^{\prime \prime} \in K_{(r)} .
$$

The proof is complete.

Similarly, we have the following lemma.

Lemma $1^{\prime}$. With the notation of Lemma 1 , if $D(T) \neq 0$ then $X_{r}(T)=$ $X_{-r}\left(T^{*}\right) N_{r}^{1} X_{-r}\left(T^{* \prime}\right) y=y^{\prime} X_{-r}\left(T^{\prime}\right) N_{r}^{1} X_{-r}\left(T^{\prime \prime}\right) \quad y, y^{\prime} \in Y^{1}, T^{*}, T^{* \prime}, T^{\prime}, T^{\prime \prime} \in$ $K_{(r)}$.

COROllary 3. With the notation of Lemma 1 , if $\Pi_{r}^{0}=\Pi_{r} \cap \Pi^{0} \neq \varnothing, T \neq$ $(0,0, \ldots, 0)$ and $D(T)=0$, then $X_{-r}(T) \notin B^{1} N^{1} B^{1} ;$ if $\Pi_{r}^{0}=\varnothing$ then $X_{-r}(T) \in$ $B^{1} N^{1} B^{1} \cup B^{1}$.

Lemma 2. Let $r \in \Sigma_{1}^{*+} \cap I\left(r_{1}^{*}\right), r_{1}^{*} \in \Pi^{*}, I_{1}(r)=\left\{r_{1}, r_{2}, \ldots, r_{n}\right\}$ and $T=$ $\left(t_{1}, t_{2}, \ldots, t_{n}\right), \quad U=\left(u_{1}, u_{2}, \ldots, u_{n}\right) \in K_{(r)}$ satisfy $D(T)=0$. Then $x=$ $X_{r}(T) X_{-r}(U)$ can be expressed in the form $x=X_{r}\left(T_{0}\right) X_{-r}\left(U_{0}\right) X_{r}\left(T_{0}^{\prime}\right) y, y \in Y^{1}$, $T_{0}, T_{0}^{\prime}, U_{0} \in K_{(r)}$, satisfying $D\left(T_{0}\right) \neq 0$.

Proof. We define $K_{0}=\{t \in K \mid \bar{t}=t\}$ and $K_{0}^{*}=K_{0} \backslash\{0\}$. We write $T_{x}=$ $\left(t_{1}-x, t_{2}, \ldots, t_{n}\right)$ and $U_{x^{-1}}=\left(u_{1}-z x^{-1}, u_{2}, \ldots, u_{n}\right), z=2$ or 1 according to $r \in \Phi_{11 b}$ or $r \in \tilde{\Phi}$. If we put $x \in K_{0}^{*}$ then $T_{x}, U_{x^{-1}} \in K_{(r)}$ since $r_{1} \notin \Phi_{1 b}$. If $x \in K_{0}^{*}$ we have $D\left(T_{x}\right)=x^{2}\left(1+x^{-1}(t+\bar{t})\right)$ and $D\left(U_{x^{-1}}\right)=z^{2} x^{-2}+z x^{-1}(u+$ $\bar{u})+D(U)$. It is clear that $D\left(T_{x}\right)=0$ and $D\left(U_{x^{-1}}\right)=0$ are the equations in the unknown $x^{-1}$ with coefficients in the field $K$. Let $\left|K_{0}^{*}\right|$ be the cardinality of $K_{0}^{*}$. Clearly, we have $\left|K_{0}^{*}\right| \geqslant 4$, so there exists at least an element $c$ of $K_{0}^{*}$ such that $D\left(T_{c}\right) \neq 0$ and $D\left(U_{c-1}\right) \neq 0$.

We write $r=r_{1}$. By Corollary 1 and Lemma $1^{\prime}$ we have

$$
\begin{aligned}
x & =X_{r}(T) X_{-r}(U)=X_{r}\left(T_{c}\right) X_{r}^{1}(c) X_{-r}(U) \\
& =X_{r}\left(T_{c}\right) X_{-r}^{1}\left(z c^{-1}\right) h_{-r}^{1}\left(-z c^{-1}\right) N_{r}^{1} X_{-r}^{1}\left(z c^{-1}\right) X_{-r}(U) \\
& =X_{r}(T) X_{-r}^{1}\left(z c^{-1}\right) h_{-r}^{1}\left(-z c^{-1}\right) N_{r}^{1} X_{-r}\left(U_{c^{-1}}\right) .
\end{aligned}
$$

Since $D\left(U_{c^{-1}}\right) \neq 0$ by Lemma 1 we have

$$
\begin{aligned}
x & =X_{r}\left(T_{c}\right) X_{-r}^{1}\left(z c^{-1}\right) h_{-r}^{1}\left(z c^{-1}\right) N_{r}^{1} X_{r}\left(T^{*}\right) N_{r}^{1} X_{r}\left(T^{* \prime}\right) y^{\prime} \\
& =X_{r}\left(T_{c}\right) X_{-r}^{1}\left(z c^{-1}\right) h_{-r}^{1}\left(-z c^{-1}\right) X_{-r}\left(U_{0}^{*}\right) X_{r}\left(T^{* \prime}\right) y^{\prime} \\
& =X_{r}\left(T_{0}\right) X_{-r}\left(U_{0}\right) X_{r}\left(T_{0}^{\prime}\right) y
\end{aligned}
$$


where $y^{\prime}, y=h_{r}(-z c) y \in Y^{1}$ and $T_{0}=T_{c}, T^{*}, T^{* \prime}, U_{0}^{*} \in K_{(r)}$ satisfying $D\left(T_{0}\right)$ $\neq 0$.

Lemma 3. Let $r \in \Sigma_{1}^{*+} \cap I\left(r_{1}^{*}\right), r_{1}^{*} \in \Pi^{*}$ and $T, U, U^{\prime} \in K_{(r)}$. Then $x=$ $X_{-r}(U) X_{r}(T) X_{-r}\left(U^{\prime}\right)$ can be expressed in the form $x=y X_{r}(\tilde{T}) X_{-r}(\tilde{U}) X_{r}\left(\tilde{T}^{\prime}\right)$, $y \in Y^{1}, \tilde{U}, \tilde{T}, \tilde{T}^{\prime} \in K_{(r)}$.

Proof. We shall consider separately the various possibilities.

$I^{\circ}$. Suppose $D(T) \neq 0$. By Lemma $1^{\prime}$ we have

$$
x=X_{-r}(U) y^{\prime} X_{-r}\left(T^{\prime}\right) N_{r}^{1} X_{-r}\left(T^{\prime \prime}\right) X_{-r}\left(U^{\prime}\right)=y^{\prime} N_{r}^{1} X_{r}\left(U_{1}\right) X_{-r}\left(U_{1}^{*}\right)
$$

where $y^{\prime} \in Y^{1}, T^{\prime}, T^{\prime \prime}, U_{1}^{*}, U_{1} \in K_{(r)}$.

(a) Suppose $D\left(U_{1}\right) \neq 0$. By Lemma $1^{\prime}$ we have

$$
\begin{aligned}
& x=y^{\prime} N_{r}^{1} y^{\prime \prime} X_{-r}\left(U_{1}^{\prime}\right) N_{r}^{1} X_{-r}\left(U_{1}^{\prime \prime}\right) X_{-r}\left(U_{1}^{*}\right)=y X_{r}(\tilde{T}) X_{-r}(\tilde{U}), \\
& \quad y^{\prime \prime} \in Y^{1}, U_{1}^{\prime}, U_{1}^{\prime \prime} \in K_{(r)} .
\end{aligned}
$$

(b) Suppose $D\left(U_{1}\right)=0$. By Lemma 2 we have

$$
x=y^{\prime} N_{r}^{1} X_{r}\left(T_{0}\right) X_{-r}\left(U_{0}\right) X_{r}\left(T_{0}^{\prime}\right), \quad y_{1}^{\prime \prime}, y_{1}^{\prime \prime} \in Y^{1}, T_{0}, T_{0}^{\prime}, U_{0} \in K_{(r)},
$$

satisfying $D\left(T_{0}\right) \neq 0$. By the results of case (a) and Proposition 5 we have $x=y_{1} X_{r}\left(\tilde{T}_{1}\right) X_{-r}\left(\tilde{U}_{1}\right) X_{r}\left(T_{0}^{\prime}\right) y_{1}^{\prime \prime}=y X_{r}(\tilde{T}) X_{-r}(\tilde{U}) X_{r}\left(\tilde{T}^{\prime}\right)$,

$$
y_{1} \in Y^{1}, \tilde{T}_{1}, \tilde{U}_{1} \in k_{(r)} .
$$

$2^{\circ}$. Suppose $D(T)=0$. By Lemma 2 we have

$$
x=X_{-r}(U) X_{r}\left(T_{0}\right) X_{-r}\left(U_{0}\right) X_{r}\left(T_{0}^{\prime}\right) y^{\prime},
$$

for $y^{\prime} \in Y^{1}, U_{0}, T_{0}, T_{0}^{\prime} \in K_{(r)}$ satisfying $D\left(T_{0}\right) \neq 0$. Thus it follows from $1^{\circ}$ that $x=y_{1} X_{r}\left(\tilde{T}_{1}\right) X_{-r}\left(\tilde{U}_{1}\right) X_{r}\left(\tilde{T}_{1}^{\prime}+T_{0}^{\prime}\right) y^{\prime}=y X_{r}(\tilde{T}) X_{-r}(\tilde{U}) X_{r}\left(\tilde{T}^{\prime}\right)$, by Corollary 1(a) and Proposition 5, where $y_{1} \in Y^{1}$ and $\tilde{U}_{1}, \tilde{T}_{1}, \tilde{T}_{1}^{\prime} \in K_{(r)}$.

The proof is complete.

Corollary 4. Let $r \in \Sigma_{1}^{*+} \cap I\left(r_{1}^{*}\right), r_{1}^{*} \in \Pi^{*}, U, T \in K_{(r)}$ and $n \in N^{1}$, $b \in B^{1}$. Then $x=b n X_{r}(T) X_{-r}(U)$ can be expressed in the form $x=$ $b^{*} n^{*} X_{-r}\left(\tilde{U}^{*}\right) X_{r}\left(\tilde{T}^{*}\right), b^{*} \in B^{1}, n^{*} \in N^{1}, \tilde{T}^{*}, \tilde{U}^{*} \in K_{(r)}$.

Proof. We denote by $w$ the image of $n$ under the natural homomorphism from $N$ onto $W$ and by $s$ the root $w(r)$.

1. Suppose $s \in \Phi^{+}$. Then $s \in \Phi^{*+}$ and $x$ can be expressed in the form $x=b X_{s_{1}}\left(T_{1}\right) n X_{-r}(U), s_{1}=I(s) \cap \Sigma_{1}^{*+}, T_{1} \in K_{(r)}$. Clearly we have $b X_{s_{1}}\left(T_{1}\right)=$ $b^{*} \in B^{1}$, so the lemma follows for this case. 
2. Suppose $s \in \Phi^{-}$.

(a) Suppose $D(T) \neq 0$. By Lemma $1^{\prime}$ and Proposition 5 we have $x=$ bny' $X_{-r}\left(T^{\prime}\right) N_{r}^{1} X_{-r}\left(T^{\prime \prime}\right) X_{-r}(U)=b y X_{s_{1}}\left(T_{1}\right) n N_{r}^{1} X_{-r}\left(\tilde{U}^{*}\right)$ where $s_{1}=I(-s) \cap$ $\Sigma_{1}^{*+}, y, y^{\prime} \in Y^{1}$ and $T^{\prime}, T^{\prime \prime} \in K_{(r)}$, so the lemma follows for this case.

(b) Suppose $D(T)=0$. By Lemma 2 we have $x=b n X_{r}\left(T_{0}\right) X_{-r}\left(U_{0}\right) X_{r}\left(T_{0}^{\prime}\right) y^{\prime}$, $y^{\prime} \in Y^{1}, T_{0}, T_{0}^{\prime}, U_{0} \in K_{(r)}$ satisfying $D\left(T_{0}\right) \neq 0$. Thus by case 2(a) and Proposition 5 the lemma is established.

This completes the proof.

We denote by $N_{0}^{1}$ the subgroup of $N^{1}$ consisting of the elements whose image under the natural homomorphism from $N$ onto $W$ belong to $W_{0}^{1}$.

Lemma 4. Let $x=$ bnvun' where $b \in B^{1}, n, n^{\prime} \in N^{1}, v \in V^{1}$ and $u \in U^{1}$. Then $x$ can be expressed in the form $x=\tilde{b} \tilde{n} \tilde{v} \tilde{u}, \tilde{b} \in B^{1}, \tilde{n} \in N^{1}, \tilde{v} \in V^{1}, \tilde{u} \in U^{1}$.

Proof. By Proposition 4 we have $n^{\prime}=n_{1} n_{2} \cdots n_{q}$ where $n_{i} \in N_{0}^{1}$ or $n_{i}=N_{r_{i}}^{1}$, $r_{i} \in \Pi^{*}, i=1,2, \ldots, q$. Let $n^{\prime \prime}=n_{2} n_{3} \cdots n_{q}$ and let $w_{1}$ be the image of $n_{1}$ under the natural homomorphism from $N$ onto $W$.

1. Suppose $n_{1} \in N_{0}^{1}$. Then we have $w_{1} \in W_{0}^{1}$, so we have $w_{1}(s) \in I(s)$ for each $s \in \Phi^{*+}$. Thus we have $x=\tilde{b}_{1} \tilde{n}_{1} \tilde{v}_{1} \tilde{u}_{1} n^{\prime \prime}, \tilde{b}_{1} \in B^{1}, \tilde{n}_{1} \in N^{1}, \tilde{v}_{1} \in V^{1}, \tilde{u}_{1} \in U^{1}$.

2. Suppose $n_{1}=N_{r_{1}}^{1}, r_{1} \in \Pi^{*}$. Clearly there is $r \in \Sigma_{1}^{*+} \cap I\left(r_{1}\right)$. We have $N_{r}^{1}=N_{r_{1}}^{1}$ since $r \in I\left(r_{1}\right)$. By Proposition 2 and Corollary $1, x$ can be expressed in the form $x=b n X_{-r}\left(U_{1}\right) v_{1}^{*} X_{r}\left(T_{1}\right) u_{1} N_{r}^{1} n^{\prime \prime}$ where $U_{1}, T_{1} \in K_{(r)}$ and $v_{1}^{*} \in V^{1}$ and $u_{1} \in U^{1}$ satisfying $-r \notin J\left(v_{1}^{*}\right)$ and $r \notin J\left(u_{1}\right)$. By Corollary 1 we have $x=b n X_{-r}\left(U_{1}\right) X_{r}\left(T_{1}\right) v_{1} u_{1} N_{r}^{1} n^{\prime \prime}=b n N_{r}^{1} X_{r}\left(U_{1}^{\prime}\right) X_{-r}\left(T_{1}^{\prime}\right) v_{1}^{\prime} u_{1}^{\prime} n^{\prime \prime}$ where $U_{1}^{\prime}, T_{1}^{\prime} \in$ $K_{(r)}$ and $v_{1}^{\prime}, v_{1} \in V^{1}$ and $u_{1}^{\prime} \in U^{1}$, satisfying $-r \notin J\left(v_{1}\right) \cup J\left(v_{1}^{\prime}\right)$ and $r \notin J\left(u_{1}\right)$ $\cup J\left(u_{1}^{\prime}\right)$. By Corollaries 4 and 1 we have $x=b^{*} n^{*} X_{-r}\left(\tilde{U}^{*}\right) X_{r}\left(\tilde{T}^{*}\right) v_{1}^{\prime} u_{1}^{\prime} n^{\prime \prime}=$ $\tilde{b}_{1} \tilde{n}_{1} \tilde{v}_{1} \tilde{u}_{1} n^{\prime \prime}$, where $b^{*}, \tilde{b}_{1} \in B^{1}, n^{*}, \tilde{n}_{1} \in N^{1}, \tilde{v}_{1} \in V^{1}, \tilde{u}_{1} \in U^{1}, \tilde{U}^{*}, \tilde{T}^{*} \in K_{(r)}$. By repeating a finite number of times the process used above we obtain the required expression for the element $x$.

Lemma $4^{\prime}$. Let $x=$ bnvuv $v^{\prime}$ where $b \in B^{1}, n \in N^{1}, v, v^{\prime} \in V^{1}$ and $u \in U^{1}$. Then $x$ can be expressed in the form $x=\tilde{b}_{0} \tilde{n}_{0} \tilde{v}_{0} \tilde{u}_{0}$ where $\tilde{b}_{0} \in B^{1}, \tilde{n}_{0} \in N^{1}, \tilde{v}_{0} \in V^{1}$ and $\tilde{u}_{0} \in U^{1}$.

Proof. By Proposition 3, $v^{\prime}$ can be expressed in the form $v^{\prime}=$ $X_{-\delta_{1}}\left(U_{1}\right) X_{-\delta_{2}}\left(U_{2}\right) \cdots X_{-\delta_{m}}\left(U_{m}\right), \delta_{j} \in \Sigma_{1}^{*+}, U_{j} \in K_{\left(\delta_{j}\right)}, j=1,2, \ldots, m, \delta_{1}<\delta_{2}$ $<\cdots<\delta_{m}$. It follows from Proposition 2(c) that there exist $w_{1} \in W^{1}$ and $r_{1} \in \Pi^{*}$ such that $w_{1}\left(\delta_{1}\right) \in I\left(r_{1}\right)$. Clearly, by Proposition 4 there exists $n_{1} \in N^{1}$ whose image under the natural homomorphism from $N$ onto $W$ is $w_{1}^{-1}$, so we have $X_{-\delta_{1}}\left(U_{1}\right)=n_{1} X_{-r}\left(T_{1}\right) n_{1}^{-1}$ where $r \in \Sigma_{1}^{*+} \cap I\left(r_{1}\right), r_{1} \in \Pi^{*}$. It follows from 
Lemma 4 that $x=\tilde{b} \tilde{n} \tilde{v} \tilde{u} X_{-r}\left(T_{1}\right) n_{1}^{-1} v^{\prime \prime}$ where $\tilde{b} \in B^{1}, \tilde{n} \in N^{1}, \tilde{v} \in V^{1}, \tilde{u} \in U^{1}$ and $v^{\prime \prime}=X_{-\delta_{2}}\left(U_{2}\right) \cdots X_{-\delta_{m}}\left(U_{m}\right) \in V^{1}$. By Corollary 1 we have $x=$ $\tilde{b} \tilde{n} X_{-r}(U) X_{r}(T) v_{1}^{\prime} u_{1}^{\prime} X_{-r}\left(T_{1}\right) n_{1}^{-1} v^{\prime \prime}$ where $U, T \in K_{(r)}$, and $v_{1}^{\prime} \in V^{1}$ and $u_{1}^{\prime} \in U^{1}$ satisfy $-r \notin J\left(v_{1}^{\prime}\right)$ and $r \notin J\left(u_{1}^{\prime}\right)$. Thus it follows from Corollary 1 that $x=$ $\tilde{b} \tilde{n} X_{-r}(U) X_{r}(T) X_{-r}\left(T_{1}\right) v_{1} u_{1} n_{1}^{-1} v^{\prime \prime}$ where $v_{1} \in V^{1}$ and $u_{1} \in U_{1}$ satisfy $-r \notin J\left(v_{1}\right)$ and $r \notin J\left(u_{1}\right)$. Thus we have

$$
\begin{aligned}
x & =\tilde{b} \tilde{n} y X_{r}(\tilde{T}) X_{-r}(\tilde{U}) X_{r}\left(\tilde{T}^{\prime}\right) v_{1} u_{1} n_{1}^{-1} v^{\prime \prime} \\
& =b^{*} n^{*} X_{-r}\left(\tilde{U}^{*}\right) X_{r}\left(\tilde{T}^{*}\right) X_{r}\left(\tilde{T}^{\prime}\right) v_{1} u_{1} n_{1}^{-1} v^{\prime \prime} \\
& =b^{*} n^{*} v_{1}^{\prime \prime} u_{1}^{\prime \prime} n_{1}^{-1} v^{\prime \prime} \\
& =\tilde{b}_{1} \tilde{n}_{1} \tilde{v}_{1} \tilde{u}_{1} v^{\prime \prime}
\end{aligned}
$$

where $y \in Y^{1}, b^{*}, \tilde{b}_{1} \in B^{1}, n^{*}, \tilde{n}_{1} \in N^{1}, v_{1}^{\prime \prime}, \tilde{v}_{1} \in V^{1}, u_{1}^{\prime \prime}, \tilde{u}_{1} \in U^{1}$ and $\tilde{T}, \tilde{U}$, $\tilde{T}^{\prime}, \tilde{U}^{*}, \tilde{T}^{*} \in K_{(r)}$. By repeating a finite number of times the process used above we obtain the required expression for the element $x$.

By Proposition 4 for each $w \in W^{1}$ we can choose an element $n_{w}$ of $N^{1}$ such that $n_{w}$ corresponds to $w$ under the natural homomorphism from $N$ onto $W$. The elements $n_{w}$, for all $w \in W^{1}$, form a set $N_{e}^{1}$.

We define $G_{1}=\cup_{w \in W^{1}} B^{1} n_{w} V^{1} B^{1}, n_{w} \in N_{e}^{1}$.

THEOREM 1. $G_{1}$ is a subgroup of $G$ and $G^{1}=G_{1}$.

Proof. If $x \in G_{1}$ then $x$ can be expressed in the form $x=b n_{w} v b^{\prime}, b^{\prime}, b \in B^{1}$, $n_{w} \in N^{1}, w \in W^{1}, v \in V^{1}$. It is clear that $x^{-1}=b^{-1} v^{-1} n_{w}^{-1} b^{-1}$. By Lemma 4 we have $x^{-1}=\tilde{b} \tilde{n} \tilde{u} \tilde{u} b^{-1}=\tilde{b} \tilde{n} \tilde{v} \tilde{b}^{\prime}$ where $\tilde{u} \in U^{1}, \tilde{b}, \tilde{b}^{\prime} \in B^{1}, \tilde{n} \in N^{1}$ and $\tilde{v} \in V^{1}$. Let $w_{1}$ denote the image of $\tilde{n}$ under the natural homomoprnism from $N$ onto $W$. Since $\tilde{n} \in N^{1}$ we have $w_{1} \in W^{1}$, so we have $\tilde{n}=h n_{w_{1}}, h \in H^{1}, n_{w_{1}} \in N_{e}^{1}$ by Proposition 4. It follows that $x^{-1} \in G_{1}$.

Let $x_{1}, x_{2} \in G_{1}$. Then $x_{1}$ and $x_{2}$ can be expressed in the form $x_{1}=b_{1} n_{w_{1}} v_{1} b_{1}^{\prime}$ and $x_{2}=b_{2} n_{w_{2}} v_{2} b_{2}^{\prime}$ where $b_{1}, b_{1}^{\prime}, b_{2}, b_{2}^{\prime} \in B^{1}, n_{w_{1}}, n_{w_{2}} \in N_{e}^{1}, w_{1}, w_{2} \in W^{1}$ and $v_{1}, v_{2} \in V^{1}$. Clearly, we have $b_{1}^{\prime} b_{2}=y u, y \in Y^{1}, u \in U^{1}$. Thus $x_{1} x_{2}$ can be expressed in the form

$$
\begin{aligned}
x_{1} x_{2} & =b_{1} n_{w_{1}} v_{1} y u n_{w_{2}} v_{2} b_{2}^{\prime}=b_{1} y^{\prime} n_{w_{1}} v_{1}^{\prime} u n_{w_{2}} v_{2} b_{2}^{\prime} \\
& =\tilde{b} \tilde{n} \tilde{v} \tilde{u} v_{2} b_{2}^{\prime} \\
& =\tilde{b}_{0} \tilde{n}_{0} \tilde{v}_{0} \tilde{u}_{0} b_{2}^{\prime}=\tilde{b}_{0} \tilde{n}_{0} \tilde{v}_{0} \tilde{b}_{0}^{\prime}
\end{aligned}
$$

(by Proposition 5)

(by Lemma 4)

where $y^{\prime} \in Y^{1}, \tilde{u}, \tilde{u}_{0} \in U^{1}, \tilde{b}, \tilde{b}_{0}, \tilde{b}_{0}^{\prime}=\tilde{u}_{0} b_{2}^{\prime} \in B^{1}$ and $v_{1}^{\prime}, \tilde{v}, \tilde{v}_{0} \in V^{1}$. It is clear that $\tilde{n}_{0}=h n_{w}$ where $h \in H^{1}$ and $n_{w} \in N_{e}^{1}, w$ being the iamge of $\tilde{n}_{0}$ under the natural homomorphism from $N$ onto $W$, so we have $x_{1} x_{2} \in G_{1}$. It follows that $G_{1}$ is a subgroup of $G$. 
For each $w \in W^{1}$ we have $B^{1} n_{w} V^{1} B^{1} \subset G^{1}$ since $B^{1}, V^{1} \subset G^{1}$ and $n_{w} \in N_{e}^{1} \subset$ $G^{1}$. Thus we have $G_{1} \subseteq G^{1}$. On the other hand, we have $U^{1}, V^{1} \subset G_{1}$. Since $G_{1}$ is a subgroup of $G$ and $G^{1}=\left\langle U^{1}, V^{1}\right\rangle$ we have $G^{1} \subseteq G_{1}$, so we have $G^{1}=G_{1}$.

By Theorem 1 and Proposition 4(c) we have

$$
G^{1}=\bigcup_{w \in W^{1}} B^{1} n_{w} V^{1} B^{1}=B^{1} N^{1} V^{1} B^{1}, \quad n_{w} \in N_{e}^{1} .
$$

The decomposition (3.A) of $G^{1}$ is called the "quasi-Bruhat" decomposition of $G^{1}$ (of $L\left(\Pi^{\theta}, K\right)$ ). In general, if $\Pi^{0} \neq \varnothing, G^{1}$ cannot be expressed in the form $G^{1}=B^{1} N^{1} B^{1}$ by Corollary 3 .

\section{The simplicity of $L\left(\Pi^{\theta}, K\right)$}

The steps of the proof of the simplicity of $L\left(\Pi^{\theta}, K\right)$ are quite analogous to those in the proof for the twisted groups given in [6].

We shall show that there exists $\lambda \in K^{*}$ such that $\lambda^{2} \neq \bar{\lambda}^{2}$ if $K^{*} \neq K_{0}^{*}$. Assuming the contrary, then $x^{2}=\bar{x}^{2}$ for all $x \in K^{*}$, so we have $(a+x)^{2}=$ $(a+\bar{x})^{2}$ where $a$ is an arbitrary element of $K_{0}^{*}$, and hence we have $x=\bar{x}$ for all $x \in K^{*}$. We have a contradiction. Thus there is $\lambda \in K^{*}$ as required. Similarly, we shall show that there exists $\mu \in K^{*}$ such that $\mu^{4} \neq \bar{\mu}^{4}$ if $K^{*} \neq K_{0}^{*}$. Assuming the contrary, we have $x^{4}=\bar{x}^{4}$ for all $x \in K^{*}$, so we have $(a \pm x)^{4}=(a \pm \bar{x})^{4}$ where $a$ is an arbitrary element of $K_{0}^{*}$, and hence we have $x^{2}=\bar{x}^{2}$ for all $x \in K^{*}$. We have a contradiction. Thus there exists $\mu \in K^{*}$ as required.

LEMMA 5. Let $r_{1}, r_{2} \in \Phi^{*+}$ such that $r_{1} \neq r_{2}$ and $r_{1} \neq \bar{r}_{2}$. Then there exists $h(\chi) \in H^{1}$ such that $\chi\left(r_{1}\right)=1, \chi\left(r_{2}\right) \neq \chi\left(r_{1}\right)$ or $\chi\left(r_{2}\right)=1, \chi\left(r_{1}\right) \neq \chi\left(r_{2}\right)$.

PROOF. We have $A_{r^{\prime} s^{\prime}}=2\left(r^{\prime}, s^{\prime}\right) /\left(r^{\prime}, r^{\prime}\right)=z\left(A_{r s}+A_{\bar{r} s}\right), r, s \in \Phi^{*}$ where $z=$ 1 or 2 according to $r \in \tilde{\Phi}$ or $r \in \Phi_{11 b}$, since $(r, \bar{r})=0$ if $r \in \Phi_{11 a}$ and $(r, \bar{r})=$ $-\frac{1}{2}(r, r)$ if $r \in \Phi_{11 b}$. Thus the following statement (4.A) holds.

(4.A) Let $h(\chi)=h_{r}^{1}(c), r \in \Phi^{*+}, c \in K_{0}$. Then for $s \in \Phi^{*}, \chi(s)=c^{\varphi\left(r^{\prime}, s^{\prime}\right)}$ where $\varphi\left(r^{\prime}, s^{\prime}\right)=z^{-1} A_{r^{\prime} s^{\prime}}$.

Let $F$ denote the set $\left\{r_{1}, r_{2}\right\}$. We shall consider separately different possibilities.

I. Suppose $I\left(r_{1}\right) \neq I\left(r_{2}\right)$.

(a) Suppose $\left(r_{1}^{\prime}, r_{2}^{\prime}\right)=0$. Then we put $h(\chi)=h_{r_{2}}^{1}(2)$. By (4.A) we have $\chi\left(r_{1}\right)=1$ and $\chi\left(r_{2}\right)=4$ or 2 according to $r_{2} \in \tilde{\Phi}$ or $r_{2} \in \Phi_{11 b}$.

(b) Suppose $\left(r_{1}^{\prime}, r_{2}^{\prime}\right) \neq 0$. (1) $F \cap \Phi_{11 b}=\varnothing$. Then we put $h(\chi)=h_{r}^{1}(-1)$ where $r \in F$ satisfies $\left(r^{\prime}, r^{\prime}\right) \geqslant\left(s^{\prime}, s^{\prime}\right), s \in F \backslash\{r\}$. It follows from the properties of Satake diagrams that $A_{r^{\prime} s^{\prime}}=\delta, \delta= \pm 1$ if $\left(r^{\prime}, r^{\prime}\right) \geqslant\left(s^{\prime}, s^{\prime}\right)$. By (4.A) we have 
$\chi(r)=(-1)^{2}=1$ and $\chi(s)=(-1)^{\delta}=-1$. (2) If there is a root $r$ of $F$ such that $r \notin \Phi_{11 b}$ and $s=F \backslash\{r\} \in \Phi_{11 b}$ then we put $h(\chi)=h_{r}^{1}(-1)$. It follows from the properties of the Satake diagram AIII that $\left(r^{\prime}, r^{\prime}\right) \geqslant\left(s^{\prime}, s^{\prime}\right)$. By (4.A) we have $\chi(r)=1$ and $\chi(s)=-1$. (3) If $F \subset \Phi_{11 b}$ then we put $h(\chi)=h_{r_{2}}^{1}(-1)$. It follows from the properties of the Satake diagram AIII that $A_{r_{2} r_{1}}=-A_{\bar{r}_{2} r_{1}}=-A_{r_{2} \bar{r}_{2}}=1$ and $A_{r_{2} r_{2}}=A_{\bar{r}_{2} \bar{r}_{2}}=2$. Thus by (4.A) we have $\chi\left(r_{1}\right)=1$ and $\chi\left(r_{2}\right)=-1$.

II. Suppose $I\left(r_{1}\right)=I\left(r_{2}\right)$.

(a) Suppose $r_{1} \notin \Phi_{11 b}$. Then we put $h(\chi)=h_{r}^{1}(\delta)$ where $r \in \Phi_{11} \cap F$, and $\delta=b^{-1} \lambda^{2}, \lambda \in K^{*}$, satisfying $\lambda^{2} \neq \bar{\lambda}^{2}$ and $b=\lambda \bar{\lambda}$. (In this case, there is $r \in F$ such that $r \in \Phi_{11}$.) It follows from the properties of the Satake diagrams that $A_{r s}=A_{\bar{r} s}=1$ and $A_{\bar{r} r}=0, s \in F \backslash\{r\}$. Thus we have $\chi(s)=b^{-2} \lambda^{2} \bar{\lambda}^{2}=1$ and $\chi(r)=b^{-2} \lambda^{4}=\lambda^{2} \bar{\lambda}^{-2} \neq 1$.

(b) Suppose $r_{1} \in \Phi_{11 b}$. (1) If there is $r \in F$ such that $r \in \Phi_{1 b}$ then we put $h(\chi)=h_{r}^{1}(-1)$. It follows from the properties of the Satake diagram AIII that $A_{r s}=1$, and hence we have $\chi(s)=-1$ and $\chi(r)=1, s \in F \backslash\{r\}\left(s \in \Phi_{11 b}\right)$. (2) If $F \cap \Phi_{1 b}=\varnothing$ then we put $h(\chi)=h_{r_{1}}^{1}(\mu) h_{\gamma}(b) h_{r_{2}}^{1}\left(\mu^{-3}\right)$, for $\gamma=r_{1}+\bar{r}_{1}$, $\mu \in K^{*}$ satisfying $\mu^{4} \neq \bar{\mu}^{4}, b=\mu \bar{\mu}$. It follows from the properties of the Satake diagram AIII that $A_{r_{1} \dot{r}_{1}}=A_{r_{2} \bar{r}_{2}}=-1$ and $A_{r_{1} \bar{r}_{2}}=0$, and $A_{\gamma r_{1}}=A_{\gamma \dot{r}_{1}}=A_{r_{1} r_{2}}=$ $A_{\gamma r_{2}}=A_{\gamma \bar{r}_{2}}=1$. Thus we have $\chi\left(r_{1}\right)=\mu^{2} \bar{\mu}^{-1} b \mu^{-3}=\mu^{2} \bar{\mu}^{-1} \mu \bar{\mu} \mu^{-3}=1$ and $\chi\left(r_{2}\right)$ $=\mu b \mu^{-6} \bar{\mu}^{3}=\mu^{2} \bar{\mu} \mu^{-6} \bar{\mu}^{3}=\mu^{-4} \bar{\mu}^{4} \neq 1$.

The proof is complete.

Obviously, such $h(\chi)$ in case (1) in the proof of Lemma 5, satisfy $\chi(\beta)=1$ for all $\beta \in \Phi^{0}$. Thus we have

Corollary 4. Let $r, s \in \Phi^{*+}$ and $r^{\prime} \neq s^{\prime}$. Then there exists $h(\chi) \in H^{1}$ such that $\chi(r)=1, \chi(s) \neq 1$ or $\chi(s)=1, \chi(r) \neq 1$, and moreover, $\chi(\beta)=1$ for all $\beta \in \Phi^{0}$.

Corollary 5. Let $n \in N^{1} \backslash N_{0}^{1}$. Then there exists $h(\chi) \in H^{1}$ such that $n h(\chi) n^{-1}=h^{\prime}\left(\chi^{\prime}\right) \neq h(\chi)$, and moreover, $\chi(\beta)=\chi^{\prime}(\beta)=1$ for all $\beta \in \Phi^{0}$.

Proof. Let $w$ denote the image of $n$ under the natural homomorphism from $N$ onto $W$. Since $n \in N^{1} \backslash N_{0}^{1}$ we have $w \in W^{1} \backslash W_{0}^{1}$. Thus by Proposition 2(a) there is $r \in \Phi^{*+}$ such that $s^{\prime}=w\left(r^{\prime}\right) \neq r^{\prime}$. By Proposition 4 we have $\rho_{\theta}^{\prime} w=w \rho_{\theta}^{\prime}$. Thus if $s=w^{\prime}(r)$ then we have $\bar{s}=w(\bar{r})$, so we have $s^{\prime}=w(r)^{\prime} \neq r^{\prime}$. We shall consider separate cases.

I. Suppose $w(r)^{\prime}=-r^{\prime}$.

(a) Suppose $r \in \Phi_{11 b}$. Then we put $h(\chi)=h_{r_{1}}^{1}$ (2) where $r_{1}=r+\bar{r} \in \Phi_{1 b}$. It is clear that $h^{\prime}\left(\chi^{\prime}\right)=h_{-r_{1}}^{1}(2)$, so we have $\chi(r)=2$ and $\chi^{\prime}(r)=\frac{1}{2}$. Thus we have $h(\chi) \neq h^{\prime}\left(\chi^{\prime}\right)$. 
(b) Suppose $r \in \tilde{\Phi}$. (1) Suppose the $\mathbb{R}$-rank of (I, $\theta$ ) is not 1 . Then it follows from the properties of the Satake diagrams that there is $r_{1} \in \Phi^{*}, I\left(r_{1}\right) \neq I(r)$, such that $\left(r^{\prime}, r_{1}^{\prime}\right) \neq 0$. We put $h(\chi)=h_{r}^{1}(2)$ or $h(\chi)=h_{r_{1}}^{1}(2)$ according to $\left(r^{\prime}, r^{\prime}\right)$ $\geqslant\left(r_{1}^{\prime}, r_{1}^{\prime}\right)$ or $\left(r^{\prime}, r^{\prime}\right)<\left(r_{1}^{\prime}, r_{1}^{\prime}\right)$. Thus we have $\chi\left(r_{1}\right)=\delta, \chi^{\prime}\left(r_{1}\right)=\delta^{-1}, \delta=2$ or $\frac{1}{2}$, or $\chi(r)=\gamma \cdot \chi^{\prime}(r)=\gamma^{-1}, \gamma=2$ or $\frac{1}{2}$, according to $\left(r^{\prime}, r^{\prime}\right) \geqslant\left(r_{1}^{\prime}, r_{1}^{\prime}\right)$ or $\left(r^{\prime}, r^{\prime}\right)<$ $\left(r_{1}^{\prime}, r_{1}^{\prime}\right)$. (2) Suppose the $\mathbb{R}$-rank of $(\Pi, \theta)$ is equal to 1 . We put $h(\chi)=h_{r}^{1}(2)$. Clearly, we have $h^{\prime}\left(\chi^{\prime}\right)=h_{-r}^{1}(2)$, so we have $\chi(r) \neq \chi^{\prime}(r)$ since $p>5$.

II. Suppose $w(r)^{\prime} \neq-r^{\prime}$. Then it follows from Corollary 4 that there is $h(\chi) \in H^{1}$ such that $\chi(r) \neq \chi(\xi s)$ where $\xi=1$ or -1 as $s \in \Phi^{*+}$ or $s \in \Phi^{*-}$. Thus we have $h(\chi) \neq h^{\prime}\left(\chi^{\prime}\right)$.

It is clear that such $h(\chi)$ above satisfy $\chi(\beta)=1$ for all $\beta \in \Phi^{0}$.

The proof is complete.

We shall prove the following statement.

(4.B) Let $h(\chi) \in H^{1}$ be such that $h(\chi) \neq 1$; then there exists $r \in \Phi^{*+}$ such that $\chi(r) \neq 1$.

Proof. Assume the contrary. Then there exists $\alpha \in \Pi^{0}$ such that $\chi(\alpha) \neq 1$. It is easy to see that there exists $s \in \Phi^{*+}$ such that $(\alpha, s) \neq 0$ so we have $s+j \alpha \in \Phi$, $j= \pm 1$. Clearly, we have $r=s+j \alpha \in \Phi^{*+}$. Thus it follows that $\chi(r) \neq 1$, $r \in \Phi^{*+}$. We have a contradiction, so the statement is established.

Lemma 6. Suppose $y \in Y^{1}$ and $y \neq 1$. Then

(1) $y \notin Z^{1}$ where $Z^{1}$ is the centre of $G^{1}$,

(2) there exists $u \in U^{1}$ such that yuy ${ }^{-1} u^{-1}=u^{*} \neq 1$ or there exists $v \in V^{1}$ such that $y v y^{-1} v^{-1}=v^{*} \neq 1$.

Proof. It is clear that statement (1) and statement (2) are equivalent. We shall prove statement (1).

Since $y \in Y^{1} \subset G_{0}$, by 8.4.4 of [1], $y$ can be expressed uniquely in the form:

$$
y=x_{\alpha_{1}}\left(t_{1}\right) x_{\alpha_{2}}\left(t_{2}\right) \cdots x_{\alpha_{p}}\left(t_{p}\right) h\left(x_{0}\right) n_{w} x_{\beta_{1}}\left(t_{1}^{\prime}\right) x_{\beta_{2}}\left(t_{2}^{\prime}\right) \cdots x_{\beta_{q}}\left(t_{q}^{\prime}\right)
$$

where $\alpha_{i}, \quad \beta_{j} \in \Phi^{0+}=\Phi^{0} \cap \Phi^{+}, t_{i}, t_{j}^{\prime} \in K^{*}, i=1,2, \ldots, p, j=1,2, \ldots, q$, $h\left(\chi_{0}\right) \in H$ and $w \in W_{0}$ satisfy $w\left(\beta_{j}\right) \in \Phi^{-}, j=1,2, \ldots, q$. We write $\Phi_{y}=$ $\left\{\alpha_{1}, \alpha_{2}, \ldots, \alpha_{p}\right\}$ and $\Phi_{y}^{\prime}=\left\{\beta_{1}, \beta_{2}, \ldots, \beta_{q}\right\}$. Suppose $\Phi_{y} \neq \varnothing$. Then there exist $\alpha \in \Phi_{y}$ and $w_{1} \in W_{0}$ such that $w_{1}(\alpha)=\beta, \beta \in \Pi^{0}$. It follows from the properties of the Satake diagrams that there exists $r \in \Phi_{11}$ such that $(r, \beta) \neq 0$. Thus we have $(s, \alpha) \neq 0, s=w_{1}^{-1}(r) \in \Phi_{11}$. Let $A_{s \alpha}=c$. Clearly, we have $c=1,-1,2$, -2. Since there exists $\lambda \in K^{*}$ such that $\lambda^{2} \neq \bar{\lambda}^{2}$ then there exists $\delta \in K^{*}$ such that $\left(\delta \bar{\delta}^{-1}\right)^{c} \neq 1$. We put $h_{1}\left(\chi_{1}\right)=h_{s}^{1}(\delta)$; hence $h_{1}\left(\chi_{1}\right) \in H^{1}$ satisfies $\chi_{1}(\alpha)=$ $\left(\delta \bar{\delta}^{-1}\right)^{c} \neq 1$. Thus we have $y \notin Z^{1}$ if $\Phi_{y} \neq \varnothing$. Similarly, we have $y \notin Z^{1}$ if $\Phi_{y}^{\prime} \neq \varnothing$. Thus the lemma is true if $\Phi_{y} \neq \varnothing$ or $\Phi_{y}^{\prime} \neq \varnothing$. Assume $\Phi_{y}=\Phi_{y}^{\prime}=\varnothing$. Then we have $y=h\left(\chi_{0}\right) n_{w}$. Suppose $w \neq 1$. Then there exists $r \in \Phi_{11}$ such that $w(r)=s \neq r$. We put $t=1$ if $\chi_{0}(s) \neq 1$, and $t \in K_{0}^{-}=\left\{t \in K^{*} \mid t \neq \bar{t}\right\}$ if 
$\chi_{0}(s)=1$. For such $t$, we have $X_{r}^{1}(t) y X_{r}^{1}(-t) \neq y$ if $y=h\left(\chi_{0}\right) n_{w}$ and $w \neq 1$. Suppose $\Phi_{y}=\Phi_{y}^{\prime}=\varnothing$ and $w=1$. Then we have $y=h\left(\chi_{0}\right) \neq 1$. Thus there exists $r \in \Phi^{*}$ such that $\chi_{0}(r) \neq 1$ by (4.B). Hence we have $y \notin Z^{1}$ if $y \neq 1$. This completes the proof.

We shall denote by $R^{1}$ an arbitrary normal subgroup of $G^{1}$ satisfying $\left|R^{1}\right|>1$. (For a set $S$, we denote by $|S|$ the cardinality of the set $S$.)

\section{LEMMA 7. $\left|R^{1} \cap U^{1}\right|>1$.}

Proof. Since $\left|R^{1}\right|>1$ there is $x^{\prime} \in R^{1}$ such that $x^{\prime} \neq 1$. By Theorem $1, x^{\prime}$ can be expressed in the form $x^{\prime}=b^{\prime} n^{\prime} v^{\prime} b^{\prime \prime}, b^{\prime}, b^{\prime \prime} \in B^{1}, n^{\prime} \in N^{1}, v^{\prime} \in V^{1}$. Since $R^{1}$ is a normal subgroup of $G^{1}$ there exists $x \in R^{1}$ such that $x \neq 1$ and $x$ can be expressed in the form $x=v b n_{w}, v \in V^{1}, b \in B^{1}, w \in W^{1}, n_{w} \in N_{e}^{1}$. We have $b=u y, u \in U^{1}, y \in Y^{1}$, so we have $x=v u y n$, where $u, v, y$ and $n_{w}=n$ are given as above. We shall consider separate cases.

(1) Suppose $u=1$ and $v=1$.

(a) Suppose $w \in W^{1} \backslash W_{0}^{1}$. Then by Corollary 5 there exists $h(\chi) \in H^{1}$ such that $n h(\chi) n^{-1}=h^{\prime}\left(\chi^{\prime}\right) \neq h(\chi)$ and $\chi(\beta)=\chi^{\prime}(\beta)=1$ for all $\beta \in \Phi^{0}$. Let $x_{1}=$ $h(\chi) x h(\chi)^{-1} x^{-1}$. Then $x_{1}=h(\chi) h^{\prime}\left(\chi^{\prime}\right)^{-1}=h_{1}\left(\chi_{1}\right) \neq 1$. Since $R^{1}$ is a normal subgroup of $G^{1}$ we have $x_{1}=h_{1}\left(\chi_{1}\right) \in R^{1}$. It follows from (4.B) that there exists $r \in \Phi^{*+}$ such that $\chi_{1}(r) \neq 1$. We define $\tilde{u}=X_{r}^{1}(t) h_{1}\left(\chi_{1}\right) X_{r}^{1}(-t) h_{1}\left(\chi_{1}\right)^{-1}, t \in$ $K_{r}^{*}$. Then we have $\tilde{u}=X_{r}^{1}\left(t^{*}\right), t^{*} \in K_{r}^{*}$ and $\tilde{u} \in R^{1}$, so the lemma is true for this case.

(b) Suppose $w \in W_{0}^{1}$. Then $x=y \in Y^{1}, y \neq 1$. By Lemma 6 there exists $u^{*} \in U^{1} \cap R^{1}$ such that $u^{*} \neq 1$ or there exists $v^{*} \in V^{1} \cap R^{1}$ such that $v^{*} \neq 1$. Obviously, the lemma is valid if there exists $u^{*} \in U^{1} \cap R^{1}, u^{*} \neq 1$. In the following (cf. case 2), we shall show that the lemma is true if there exists $v^{*} \in V^{1} \cap R^{1}, v^{*} \neq 1$.

(2) Suppose $v=1, u \neq 1$ or $v \neq 1$.

We write $s=J_{1}(u)$ or $s=J_{1}(v)$ according as $v=1$ or $v \neq 1$. Then $h(\chi)=$ $h_{s}^{1}(2)$ satisfies $\chi(s)=2$ or 4 according as $s \in \Phi_{11 b}$ or $s \in \tilde{\Phi}$. Moreover, we have $\chi(\beta)=1$ for all $\beta \in \Phi^{0}$. We define $x_{1}^{\prime}=h(\chi) x h(\chi)^{-1} x^{-1}$.

(a) Suppose $v=1, u \neq 1$. Then we have $x_{1}^{\prime}=u_{1}^{\prime} h(\chi) h^{\prime}\left(\chi^{\prime}\right)^{-1} u^{-1}=$ $u_{1}^{\prime} h_{1}\left(\chi_{1}\right) u^{-1}$ where $u_{1}^{\prime}=h(\chi) u h(\chi)^{-1} \in U^{1}$, and $h^{\prime}\left(\chi^{\prime}\right)=n h(\chi) n^{-1}$ and $h_{1}\left(\chi_{1}\right)$ $=h(\chi) h^{\prime}\left(\chi^{\prime}\right)^{-1} \in H^{1}$. We write $x_{1}=u^{-1} u_{1}^{\prime} h_{1}\left(\chi_{1}\right)$. Clearly, we have $x_{1}^{\prime}, x_{1} \in R^{1}$ since $R^{1}$ is a normal subgroup of $G^{1}$. Since $\chi(s) \neq 1$ we have $u_{1}=u^{-1} u_{1}^{\prime} \neq I$, $J_{1}\left(u_{1}\right)=s$, so $x_{1}=u_{1} h_{1}\left(\chi_{1}\right), u_{1} \in U^{1}, u_{1} \neq 1$. Let $\tilde{u}=h(\chi) x_{1} h(\chi)^{-1} x_{1}^{-1}$. Then we have $\tilde{u} \in U^{1} \cap R^{1}, \tilde{u} \neq 1$, since $\chi(s) \neq 1$ and $J_{1}\left(u_{1}\right)=s$, so the lemma follows for this case.

(b) Suppose $v \neq 1$. Similarly, then we have $x_{1}=h_{1}\left(\chi_{1}\right) v_{1} u_{1} \in R^{1}$ where $v_{1} \in$ $V^{1}, J_{1}\left(v_{1}\right)=s, v_{1} \neq 1, u_{1} \in U^{1}$ and $h_{1}\left(\chi_{1}\right) \in H^{1}$. Since $v_{1} \neq 1$ then $J\left(v_{1}\right)$ can 
be expressed in the form $J\left(v_{1}\right)=\left\{-s_{1},-s_{2}, \ldots,-s_{q}\right\}, s_{i} \in \Phi_{1}^{*+}, i=1,2, \ldots, q$, $q \geqslant 1$. Suppose $q>1$. By Lemma 5 there exists $h(\chi) \in H^{1}$ such that $\chi\left(s_{1}\right)=1$, $\chi\left(s_{2}\right) \neq 1$ or $\chi\left(s_{2}\right)=1, \chi\left(s_{1}\right) \neq 1$. We write $x_{2}^{\prime}=x_{1}^{-1} h(\chi) x_{1} h(\chi)^{-1}$. Then we have $x_{2}^{\prime}=u_{1}^{-1} v_{2} u_{1}^{*}$ where $v_{2}=v_{1}^{-1} h(\chi) v_{1} h(\chi)^{-1} \in V^{1}$ and $u_{1}^{*}=h(\chi) u_{1} h(\chi)^{-1}$. We write $x_{2}=v_{2} u_{1}^{*} u_{1}^{-1}=v_{2} u_{2}, u_{2}=u_{1}^{*} u_{1}^{-1} \in U^{1}$. By the properties of $h(\chi)$ mentioned above we have $J\left(v_{2}\right)=\left\{-s_{1}^{*},-s_{2}^{*}, \ldots,-s_{p}^{*}\right\}, s_{i}^{*} \in \Phi_{1}^{*+}, i=1,2, \ldots, p$ satisfying $-s_{1}^{*}<-s_{1}$ or $-s_{1}^{*}=-s_{1},-s_{2}^{*}<-s_{2}$ according as $\chi\left(s_{1}\right)=1, \chi\left(s_{2}\right) \neq 1$ or $\chi\left(s_{2}\right)=1, \chi\left(s_{1}\right) \neq 1$. Clearly we have $x_{2}^{\prime}, x_{2} \in R^{1}$. By repeating a finite times the process used above, finally there is $x^{*} \in R^{1}$ such that $x^{*}$ can be expressed in the form $x^{*}=v^{*} u^{*}$ where $u^{*} \in U^{1}$ and $v^{*} \in V^{1}$ satisfying $J\left(v^{*}\right)=\{-r\}$, $r \in \Phi^{*+}$, so we have $x^{*}=X_{-r}^{1}(t) u^{*}, t \in K_{r}^{*}$. This means $q=1$. It follows from Lemma 5 that $x^{*}=X_{r}^{1}\left(t^{\prime-1}\right) h_{r}^{1}\left(-t^{\prime-1}\right) N_{r}^{1} X_{r}^{1}\left(t^{\prime-1}\right) u^{*}, t^{\prime}=z^{-1} t$. We write $\tilde{x}^{*}=$ $h_{r}^{1}\left(-t^{\prime-1}\right) N_{r}^{1} X_{r}^{1}\left(t^{\prime-1}\right) u X_{r}^{1}\left(t^{\prime-1}\right)$. Since $R^{1}$ is a normal subgroup of $G^{1}$ we have $\tilde{x}^{*} \in R^{1}$. It is clear that $\tilde{x}^{*}=h_{r}^{1}\left(-t^{-1}\right) N_{r}^{1} \tilde{u}^{*}, \tilde{u}^{*} \in U^{1}$. If $\tilde{u}^{*}=1$ then the lemma is true for this case by (1a) above. If $\tilde{u}^{*} \neq 1$ then the lemma is true for this case by (2a) above. Thus the lemma is valid for the case (2b). Clearly, the cases (1a), (1b), (2a) and (2b), considered above, exhaust all possibilities. The proof is complete.

For each $r \in \Phi^{*}$ we define $X_{r}^{1}=\left\{X_{r}^{1}(t), t\right.$ runs through $\left.K_{r}\right\}$.

LEMMA 8. There exists $r \in \Phi^{*}$ such that $\left|R^{1} \cap X_{r}^{1}\right|>I$.

Proof. By Lemma 7 there exists $u \in R^{1} \cap U^{1}, u \neq 1$. We write $J(u)=$ $\left\{r_{1}, r_{2}, \ldots, r_{p}\right\}, r_{i} \in \Phi_{1}^{*+}, i=1,2, \ldots, p, p \geqslant 1$. Clearly, the lemma is valid for the case of $p=1$. Assume $p>1$. By Lemma 5, there exists $h(\chi) \in H^{1}$ such that $\chi\left(r_{1}\right)=1, \chi\left(r_{2}\right) \neq 1$ or $\chi\left(r_{2}\right)=1, \chi\left(r_{1}\right) \neq 1$. Let $u_{1}=u^{-1} h(\chi) u h(\chi)^{-1}$. It is clear that $u_{1} \in R^{1} \cap U^{1}$ and $J\left(u_{1}\right)=\left\{r_{1}^{*}, r_{2}^{*}, \ldots, r_{q}^{*}\right\}, \quad r_{i}^{*} \in \Phi_{1}^{*+}, i=$ $1,2, \ldots, q$, satisfying $r_{1}<r_{1}^{*}$ or $r_{1}=r_{1}^{*}, r_{2}<r_{2}^{*}$, according as $\chi\left(r_{1}\right)=1, \chi\left(r_{2}\right)$ $\neq 1$ or $\chi\left(r_{2}\right)=1, \chi\left(r_{1}\right) \neq 1$. Finally, by repeating a finite number of times the process used above we have $u^{*}=X_{r}^{1}(t) \in R^{1} \cap X_{r}^{1}, t \in K_{r}^{*}$. This completes the proof.

LEMMA 9. For every $s \in \Phi^{*+}, X_{s}^{1} \subset R^{1}$.

Proof. We shall prove the following statements.

(a) Let $h(\chi) \in R^{1}$ such that $\chi(r) \neq 1, r \in \Phi^{*}$. Then $X_{r}^{1} \subset R^{1}$.

For every $c \in K_{r}$ we put $t=-(1-\chi(r))^{-1} c$, and hence we have $h(\chi) X_{r}^{1}(t) h(\chi)^{-1} X_{r}^{1}(-t)=X_{r}^{1}(-(1-\chi(r)) t)=X_{r}^{1}(c) \in R^{1}$. The statement follows.

(b) Let $X_{r}^{1}(t) \in R^{1}, r \in \Phi_{11 b}^{*+}=\Phi_{11 b} \cap \Phi_{1}^{*+}, t \in K_{r}^{*}$. Then there exists $u \in$ $K_{r+\dot{r}}^{*}$ such that $X_{r+\bar{r}}^{1}(u) \in R^{1}$. 
It is clear that $\lambda t \in K_{r}=K^{*}, \lambda \in K^{*}, \lambda \neq \bar{\lambda}$. We have $X_{r}^{1}(t) X_{r}^{1}(\lambda t) X_{r}^{1}(-t) X_{r}^{1}(-\lambda t)=X_{r+\bar{r}}^{1}(u) \in R^{1}$ where $u=\eta(t \overline{\lambda t}+\lambda t \bar{t})=\eta t \bar{t}(\lambda-$ $\bar{\lambda}) \neq 0, u \in K_{r+\bar{r}}^{*}, \eta= \pm 1$. The statement follows.

(c) Let $X_{r}^{1}(t) \in R^{1}, r \in \Phi_{1}^{*+} \backslash \Phi_{11 b}^{*+}, t \in K_{r}^{*}$. Then $X_{r}^{1} \subset R^{1}$.

We have $h_{r}^{1}\left(t^{-1}\right) X_{r}^{1}(t) h_{r}^{1}\left(t^{-1}\right)^{-1}=X_{r}^{1}\left(t^{-1}\right)$ and $N_{r}^{1} X_{r}^{1}\left(t^{-1}\right)\left(N_{r}^{1}\right)^{-1}=X_{-r}^{1}\left(-t^{-1}\right)$, so we have $X_{-r}^{1}\left(-t^{-1}\right) \in R^{1}$. It follows that $N_{r}^{1}(t)=X_{r}^{1}(t) X_{-r}^{1}\left(-t^{-1}\right) X_{r}^{1}(t) \in R^{1}$ since $r \in \Phi_{1}^{*+} \backslash \Phi_{11 b}^{*+}$. It is clear that $X_{r}^{1}(2 t) \in R^{1}$. Similarly, we have $N_{r}^{1}(2 t) \in$ $R^{1}$. We write $h(\chi)=N_{r}^{1}(2 t) N_{r}^{1}(t)$. Clearly we have $h(\chi) \in R^{1}$ and $h(\chi)=$ $h_{r}^{1}(2 t) \tilde{N}_{r}^{1} h_{r}^{1}(t) \tilde{N}_{r}^{1}=h_{r}^{1}(2 t) \tilde{N}_{r}^{1} h_{r}^{1}(t)\left(\tilde{N}_{r}^{1}\right)^{-1}\left(\tilde{N}_{r}^{1}\right)^{2}=h_{r}^{1}(2 t) h_{r}^{1}\left(-t^{-1}\right) h_{r}^{1}(-1)=h_{r}^{1}(2)$ where $\tilde{N}_{r}^{1}=n_{r}(1)$ if $r \in \Phi_{1}, \tilde{N}_{r}^{1}=N_{r}^{1}$ if $r \in \Phi_{11}$. Since $r \in \Phi_{1}^{*+} \backslash \Phi_{11 b}^{*+}$ we have $\chi(r)=4 \neq 1$. Thus by statement (a) above we have $X_{r}^{1} \subset R^{1}$.

By Lemma 8 and statements (b) and (c) we obtain the following statement.

(d) There exists $r \in \Phi_{1}^{*+} \backslash \Phi_{11 b}^{*+}$ such that $X_{r}^{1} \subset R^{1}$.

By a process which is similar to that in the proof of statement (c) we have the following statement immediately.

(e) Let $X_{r}^{1} \subset R^{1}, r \in \Phi_{1}^{*+} \backslash \Phi_{11 b}^{*+}$. Then $h_{r}^{1}(u) \in R^{1}$ for all $u \in K_{r}^{*+}$.

Now, we shall prove the lemma.

Clearly, this lemma is true for the case of $\Pi^{0}=\varnothing$. In the following we assume $\Pi^{0} \neq \varnothing$. By statements (d) and (e) there exists $r \in \Phi_{1}^{*+} \backslash \Phi_{11 b}^{*+}$ such that $h_{r}^{1}(u) \in R^{1}$ for all $u \in K_{r}^{* \prime}$. It is clear that there exist elements $\lambda_{1}, \lambda_{2}, \lambda_{3}$ and $\lambda_{4}$ of $K^{*}$ satisfying condition

(4.C): $\left(\lambda_{1} \bar{\lambda}_{1}\right)^{2} \neq 1, \lambda_{2}^{2} \neq \bar{\lambda}_{2}^{2}, \lambda_{3}^{2} \bar{\lambda}_{3} \neq 1$ and $\lambda_{4}^{2} \neq \bar{\lambda}_{4}$.

All such elements $\lambda_{1}, \lambda_{2}, \lambda_{3}$ and $\lambda_{4}$ of $K^{*}$ satisfying (4.C) form a subset $S$ of $K^{*}$. If $r=\bar{r}$ we put $h(\chi)=h_{r}^{1}(2)$, so we have $\chi\left(s^{*}\right) \neq 1$ for each $s^{*} \in \Phi^{*+}$, satisfying $\left(r, s^{*}\right) \neq 0$ since $A_{r s^{*}}=1,-1,2$ or -2 . If $r \neq \bar{r}$ and $s^{*} \in \Phi^{*+}$, satisfy $\left(r, s^{*}\right) \neq 0$ or $\left(\bar{r}, s^{*}\right) \neq 0$ then we have $A_{r s^{*}}= \pm 1, \pm 2$ if $\left(r, s^{*}\right) \neq 0$ and $A_{\bar{r} s^{*}}= \pm 1, \pm 2$ if $\left(\bar{r}, s^{*}\right) \neq 0$, so we can choose an appropriate $\lambda^{*}$ of $S$ such that $h(\chi)=h_{r}^{1}\left(\lambda^{*}\right)$ satisfies $\chi\left(s^{*}\right) \neq 1$. It is clear that such $h(\chi)$ as above belongs to $R^{1}$. By Proposition 2(c), for each $s \in \Phi^{*+}$ there exists $w \in W^{1}$ such that $\left(r^{\prime}, s_{1}^{\prime}\right) \neq 0, s_{1}=w(s)$, and hence we have

$\left(r+\bar{r}, s_{1}+\bar{s}_{1}\right)=\left(r, s_{1}\right)+\left(r, \bar{s}_{1}\right)+\left(\bar{r}, s_{1}\right)+\left(\bar{r}, \bar{s}_{1}\right)=2\left(r, s_{1}\right)+2\left(\bar{r}, s_{1}\right) \neq 0$.

Hence $\left(r, s_{1}\right)$ and $\left(\bar{r}, s_{1}\right)$ cannot be equal to zero simultaneously, so we have $\left(r, s_{1}\right) \neq 0$ or $\left(\bar{r}, s_{1}\right) \neq 0$. By the results mentioned above and statement (a) we have $X_{s_{1}}^{1} \subset R^{1}$. It follows that $n X_{s_{1}}^{1} n^{-1}=X_{s}^{1} \subset R^{1}$ where $n \in N^{1}$ whose image under the natural homomorphism from $N$ onto $W$ is $w^{-1}$. This completes the proof.

By Lemma 9 we obtain the following corollary immediately.

CoRollary 6. $U^{1}, V^{1} \subset R^{1}$.

THEOREM 2. $G^{1}$ is a simple group. 
Proof. By Corollary 6 we have $U^{1}, V^{1} \subset R^{1}$, so we have $G^{1}=\left\langle U^{1}, V^{1}\right\rangle \subseteq R^{1}$. On the other hand, we have $R^{1} \subseteq G^{1}$. It follows that $R^{1}=G^{1}$, so $G^{1}$ is a simple group. This completes the proof.

\section{The identifications of $L\left(\Pi^{\theta}, K\right)$}

See Table II.

(1) If all the circles of the Satake diagram $\Pi^{\theta}$ are white, $\Pi^{\theta}$ is called a normal diagram or a quasi-normal diagram according as the Satake diagram $\Pi^{\theta}$ does not have double-ended arrows or does have double-ended arrows.

If the Satake diagram $\Pi^{\theta}$ is a normal diagram then the simple group $L\left(\Pi^{\theta}, K\right)$ is identified with the Chevalley group $L\left(K_{0}\right)$; if the Satake diagram $\Pi^{\theta}$ is a quasi-normal diagram then the simple group $L\left(\Pi^{\theta}, K\right)$ is identified with the twisted group ${ }^{2} L(K)$.

(2) Let $\Pi^{\theta}$ be a Satake diagram and let $k$ be the number of black circles of the Satake diagram $\Pi^{\theta}$.

(I) Let $\Pi^{\theta}$ be the Satake diagram of the type AIII. Then $\mathbf{A}_{n}\left(\Pi^{\theta}, K\right) \cong$ $P S U_{n+1}(K, f), \quad f=\left(\bar{x}_{1} x_{n+1}+\eta \bar{x}_{n+1} x_{1}\right)-\left(\bar{x}_{2} x_{n}+\eta x_{n} x_{2}\right)$ $+\cdots+(-1)^{m}\left(\bar{x}_{m} x_{n-m+2}+\eta \bar{x}_{n-m+2} x_{m}\right)+x_{m+1} \bar{x}_{m+1}+\cdots+x_{m+k+1} \bar{x}_{m+k+1}$, $m=\frac{1}{2}(n-k), \eta=(-1)^{n}, m$ must be a positive integer.

(II) Let $\Pi^{\theta}$ be the Satake diagram of the type BI. Then $\mathbf{B}_{n}\left(\Pi^{\theta}, K\right) \cong$ $P \Omega_{2 n+1}\left(K_{0}, f\right)$,

$$
\begin{aligned}
f= & x_{0} x_{0}+x_{1} x_{-1}+x_{2} x_{-2}+\cdots+x_{n-k} x_{-n+k} \\
& +\left(x_{n-k+1}-\alpha x_{-(n-k+1)}\right)\left(x_{n-k+1}-\bar{\alpha} x_{-(n-k+1)}\right) \\
& +\left(x_{n-k+2}-\alpha x_{-(n-k+2)}\right)\left(x_{n-k+2}-\bar{\alpha} x_{-(n-k+2)}\right)+\cdots \\
& +\left(x_{n}-\alpha x_{-n}\right)\left(x_{n}-\bar{\alpha} x_{-n}\right),
\end{aligned}
$$

$\alpha$ being a generator of $K$ over $K_{0}$.

(III) Let $\Pi^{\theta}$ be the Satake diagram of the type DI. Then $\mathbf{D}_{n}\left(\Pi^{\theta}, K\right) \cong$ $P \Omega_{2 n}\left(k_{0}, f\right)$,

$$
\begin{aligned}
f= & x_{1} x_{-1}+x_{2} x_{-2}+\cdots+x_{n-k} x_{-n+k} \\
& +\left(x_{n-k+1}-\alpha x_{-(n-k+1)}\right)\left(x_{n-k+1}-\bar{\alpha} x_{-(n-k+1)}\right) \\
& +\left(x_{n-k+2}-\alpha x_{-(n-k+2)}\right)\left(x_{n-k+2}-\bar{\alpha} x_{-(n-k+2)}\right)+\cdots \\
& +\left(x_{n}-\alpha x_{-n}\right)\left(x_{n}-\bar{\alpha} x_{-n}\right),
\end{aligned}
$$

$\alpha$ being a generator of $K$ over $K_{0}$.

The proof of this statement will be given in (A.1).

(3) If we put $K=\mathbf{C}$, then all simple groups $L\left(\Pi^{\theta}, \mathbf{C}\right)$ exhaust all simple algebraic groups defined over $\boldsymbol{R}$ which have been considered by J. Tits [7] (except the two types given by Table $\mathrm{II}^{\prime}$ ). The construction of the simple groups 
$L\left(\Pi^{\theta}, \mathbb{C}\right)$ for all Satake (except the types given by Table $\left.\mathrm{II}^{\prime}\right)$ is a uniform construction of the simple algebraic groups defined over $\mathbb{R}$ which are individually given (or constructed) for different Satake diagrams ( $\mathbb{R}$-indices) by J. Tits [7]. The detailed consideration about $L\left(\Pi^{\theta}, \mathbf{C}\right)=L(\Pi, \theta ; \mathbf{C}, f)$ will given in another paper.

(4) Suppose $K=G F\left(q^{2}\right), q$ being some prime-power. Then $L\left(\Pi^{\theta}, K\right) \cong L\left(K_{0}\right)$ or ${ }^{2} L(K)$ according as $\rho_{\theta} \in \operatorname{Ad} L$ or $\rho_{\theta} \in$ Aut $L \backslash \operatorname{Ad} L$.

We shall prove this statement for the Satake diagram $\Pi^{\theta}$ of the type AII in (A.2). For the Satake diagram of other types the proof of this statement is similar to that for the Satake diagram of type AII.

In the following we shall denote by $I_{s}$ the $s \times s$ unit matrix.

(A.1) We shall prove statement (2).

(I) If we change the matrix $A$ in 14.5.1 of [1] to the matrix $A_{(k)}$ then we obtain an argument along the lines of the proof of 14.5 .1 of [1] by which the statement (2)(I), can be proved, where $A_{(k)}$ is defined by

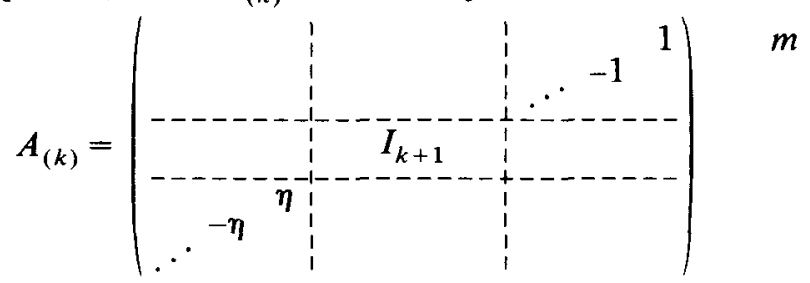

(III) If we change the matrices $B, A$ and $S$ in 14.5.2 of [1] to the form $B_{(k)}$, $A_{(k)}$ and $S_{(k)}$ respectively then we obtain an argument along the lines of the proof of 14.5 .2 of [1] by which statement 2.III can be proved, where

$$
\begin{gathered}
B_{(k)}=\left(\begin{array}{lll}
I_{2 n-2 k} & \\
& B_{0}^{(k)}
\end{array}\right), \quad A_{(k)}=\left(\begin{array}{lll}
I_{n-k} & & \\
& & A_{0}^{(k)}
\end{array}\right), \\
S_{(k)}=\left(\begin{array}{lll}
I_{2 n-2 k} & \\
& S_{0}^{(k)}
\end{array}\right), \\
\\
\end{gathered}
$$


The matrices $B_{0}, A_{0}$ and $S_{0}$ are given in 14.5.2 of [1] and the number of the matrices $B_{0}, A_{0}$ and $S_{0}$ within the matrices $B_{0}^{(k)}, A_{0}^{(k)}$ and $S_{0}^{(k)}$ are all equal to $k$. (We write the rows and columns in the order $1,2, \ldots, n-k,-1,-2, \ldots$, $-(n-k), n-k+1,-(n-k+1), \ldots, n,-n$.)

(II) By a procedure which is similar to those of the proof of 2.III mentioned above the statement 2 .II is established.

(A.2) We shall prove the statement mentioned in 5.

Since $L \cong \mathbf{A}_{n}$, the fundamental root system $\Pi$ of $L$ can be expressed in the form $\Pi=\left\{\alpha_{1}, \alpha_{2}, \ldots, \alpha_{n}\right\}, n$ being odd, $\alpha_{i}=e_{i}-e_{i+1}, i=1,2, \ldots, n$. For the Satake diagram $\Pi^{\theta}$ of type AIII we have $\rho_{\theta}^{\prime}=w_{\alpha_{1}} w_{\alpha_{3}} \cdots w_{\alpha_{n}}$ and $\rho_{\theta}=n_{\alpha_{1}} n_{\alpha_{3}}$ $\cdots n_{\alpha_{n}}$. Since $K=G F\left(q^{2}\right)$ there exists $b \in K^{*}$ such that $b \bar{b}=-1$ where $\bar{b}=b^{q}$. Let $\varepsilon$ denote the generator of the multiplicative group $K^{*}$. We define $\rho_{i}^{*}=$ $x_{\alpha_{i}}\left(a_{i}\right) x_{-\alpha_{1}}\left(b_{i}\right), a_{i}=\frac{1}{2} \bar{b}_{i}, \quad b_{i}=b^{\delta_{i}}, \delta_{i}=(-1)^{(i-1) / 2}, i=1,3, \ldots, n$, and $\rho^{*}=$ $h^{*}\left(\chi^{*}\right) \rho_{1}^{*} \rho_{3}^{*} \cdots \rho_{n}^{*}, h\left(\chi^{*}\right) \in H$ satisfying $\chi^{*}\left(\alpha_{i}\right)=-\varepsilon^{\delta_{i}}, i=1,3, \ldots, n$, and $\chi^{*}\left(\alpha_{j}\right)=1, j=2,4, \ldots, n-1$. Since $\rho_{i}^{*} \in G$ we have

$$
\begin{aligned}
& \sigma\left(\rho_{i}^{*}\right)=x_{-\alpha_{i}}\left(-\bar{a}_{i}\right) x_{\alpha_{i}}\left(-\bar{b}_{i}\right)=x_{-\alpha_{i}}\left(-\bar{a}_{i}\right) x_{-\alpha_{i}}\left(-\bar{b}_{i}^{-1}\right) h_{-\alpha_{i}}\left(\bar{b}_{i}^{-1}\right) n_{-\alpha_{i}} x_{-\alpha_{i}}\left(-\bar{b}_{i}^{-1}\right) \\
& \quad=x_{-\alpha_{i}}\left(-\bar{a}_{i}-\bar{b}_{i}^{-1}\right) h_{-\alpha_{i}}\left(\bar{b}_{i}^{-1}\right) n_{-\alpha_{i}} x_{-\alpha_{i}}\left(-\bar{b}_{i}^{-1}\right) \\
& \quad=n_{\alpha_{i}} h_{\alpha_{i}}\left(b_{i}\right) x_{\alpha_{i}}\left(\bar{b}_{i}\left(\bar{a}_{i} \bar{b}_{i}+1\right)\right) x_{-\alpha_{i}}\left(b_{i}\right)=n_{\alpha_{i}} h_{\alpha_{i}}\left(\bar{b}_{i}\right) \rho_{i}^{*}, \quad i=1,3, \ldots, n .
\end{aligned}
$$

It follows that

$$
\begin{gathered}
\sigma\left(\rho^{*}\right)=\rho_{\theta} \tilde{h}(\tilde{\chi}) \rho^{*}, \\
\tilde{h}(\tilde{\chi})=\rho_{\theta} \sigma\left(h^{*}\left(\chi^{*}\right)\right) \rho_{\theta} h^{*}\left(\chi^{*}\right)^{-1} h_{\alpha_{1}}\left(\bar{b}_{1}\right) h_{\alpha_{3}}\left(\bar{b}_{3}\right) \cdots h_{\alpha_{n}}\left(\bar{b}_{n}\right) \in H .
\end{gathered}
$$

It is clear that $\tilde{h}(\tilde{\chi})$ satisfies the relations $\tilde{\chi}\left(\alpha_{i}\right)=\left(\bar{\varepsilon} \varepsilon^{-1} \bar{b}^{2}\right)^{\delta_{i}}, i=1,3, \ldots, n$, and $\tilde{\chi}\left(\alpha_{j}\right)=\bar{b} \bar{b}^{-1}=1, j=2,4, \ldots, n+1$. Since $b \bar{b}=1$ we have $b=\varepsilon^{(q-1) / 2}$, so we have $\varepsilon^{q} \varepsilon^{-1} \bar{b}^{2}=\varepsilon^{q-1} \varepsilon^{(q-1) q}=\varepsilon^{q^{2}-1}=1$. Thus we have $\tilde{\chi}\left(\alpha_{i}\right)=1, i=1,3, \ldots, n$. It follows that $\sigma\left(\rho^{*}\right)=\rho_{\theta} \rho^{*}$.

Clearly, $L\left(K_{0}\right)=\left\langle x_{r}(t), r\right.$ runs through $\Phi, t$ runs through $\left.K_{0}\right\rangle$. For each $r \in \Phi^{*}, t \in K_{0}$, we have

$$
\begin{aligned}
\sigma\left(\rho^{*-1} x_{r}(t) \rho^{*}\right) & =\sigma\left(\rho^{*}\right)^{-1} \sigma\left(x_{r}(t)\right) \sigma\left(\rho^{*}\right) \\
& =\rho^{*-1} \rho_{\theta}^{-1} x_{\bar{r}}\left(k_{r} t\right) \rho_{\theta} \rho^{*} \\
& =\rho^{*-1} x_{r}(t) \rho^{*}
\end{aligned}
$$

Thus for $r \in \Phi^{*+}, t \in K_{0}$, we have $\rho^{*-1} x_{r}(t) \rho^{*} \in U^{1}$ and $\rho^{*-1} x_{-r}(t) \rho^{*} \in V^{1}$. 
For each $\alpha \in \Pi^{0}$ there exist $r_{1}, r_{2} \in \Phi^{*}$ such that $\alpha=r_{1}+r_{2}$. Moreover, for each $t \in K_{0}$ there exist $t_{1}, t_{2} \in K_{0}^{\prime}$ such that

$$
x_{\alpha}(t)=x_{r_{1}}\left(t_{1}\right) x_{r_{2}}\left(t_{2}\right) x_{r_{1}}\left(-t_{1}\right) x_{r_{2}}\left(-t_{2}\right) .
$$

Thus we have $\rho^{*-1} x_{\alpha}(t) \rho^{*} \in G^{1}, \alpha \in \Phi^{0}, t \in K_{0}$. It follows that $\rho^{*-1}\left(L\left(K_{0}\right)\right) \rho^{*}$ $\subset G^{1}$.

Clearly, an element $x$ of $G=L(K)$ belongs to $L\left(K_{0}\right)$ if and only if $f(x)=x$. It is clear that $f(x)=\rho_{\theta} \sigma(x) \rho_{\theta}^{-1}$ for all $x \in G$. Thus it follows from $\rho_{\theta}^{2}=1$ and $\sigma\left(\rho^{*}\right)=\rho_{\theta} \rho^{*}$ that for each $r \in \Phi^{*}, t \in K_{r}$,

$$
\begin{aligned}
f\left(\rho^{*} X_{r}^{1}(t) \rho^{*-1}\right) & =\rho_{\theta} \sigma\left(\rho^{*} X_{r}^{1}(t) \rho^{*-1}\right) \rho_{\theta}^{-1}=\rho_{\theta} \sigma\left(\rho^{*}\right) \sigma\left(X_{r}^{1}(t)\right) \sigma\left(\rho^{*-1}\right) \rho_{\theta} \\
& =\rho_{\theta} \rho_{\theta} \rho^{*-1} X_{r}^{1}(t) \rho^{*-1} \rho_{\theta} \rho_{\theta}=\rho^{*} X_{r}^{1}(t) \rho^{*-1}
\end{aligned}
$$

so we have $\rho^{*} X_{r}^{1}(t) \rho^{*-1} \in L\left(K_{0}\right)$. Thus we have $\rho^{*}\left(L\left(\Pi^{\theta}, K\right)\right) \rho^{*-1}=L\left(K_{0}\right)$. This means for all $x \in G=L(K)$ that the mapping $\psi: x \rightarrow \rho^{*-1} x \rho^{*}$ is an automorphism of $G$ and $\psi\left(L\left(K_{0}\right)\right)=L\left(\Pi^{\theta}, K\right)$. Thus we have $L\left(K_{0}\right) \cong$ $L\left(\Pi^{\theta}, K\right)$.

\section{TABLE I}

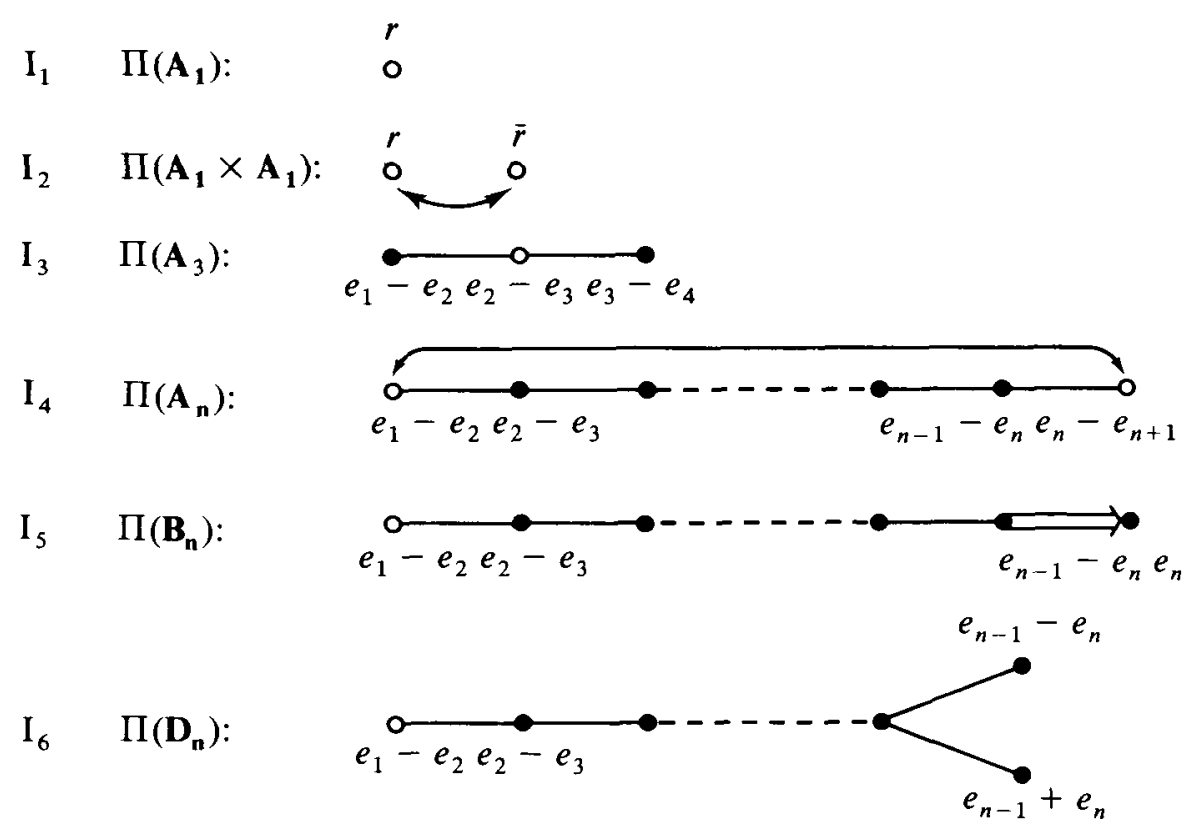


TABLE $I I_{0}$

$\Pi\left(\mathbf{A}_{n}\right):$

$$
e_{1}-e_{2} e_{2}-e_{3}-\infty-e_{n-1} e_{n}-e_{n+1}
$$

$\Phi\left(\mathbf{A}_{n}\right):$

$$
\left\{e_{i}-e_{j}, i \neq j, i, j=1,2, \ldots, n+1\right\}
$$

$\Pi\left(\mathbf{B}_{n}\right)$ :

$$
e_{1} \stackrel{\circ-e_{2} e_{2}-e_{3}}{\circ} \underset{e_{n-1}-e_{n} e_{n}}{-\infty}
$$

$\Phi\left(\mathbf{B}_{n}\right):$

$$
\left\{e_{i} \pm e_{j}, i \neq j, i, j=1,2, \ldots, n, \pm e_{i}, i=1,2, \ldots, n\right\}
$$

$\Pi\left(\mathbf{C}_{n}\right)$ :

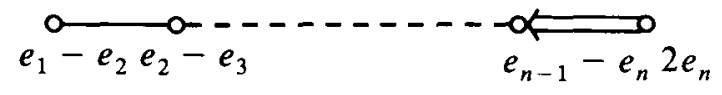

$\Phi\left(\mathbf{C}_{n}\right):$

$$
\left\{e_{i} \pm e_{j}, i \neq j, i, j=1,2, \ldots, n, \pm 2 e_{i}, i=1,2, \ldots, n\right\}
$$

$\Pi\left(\mathbf{D}_{n}\right):$

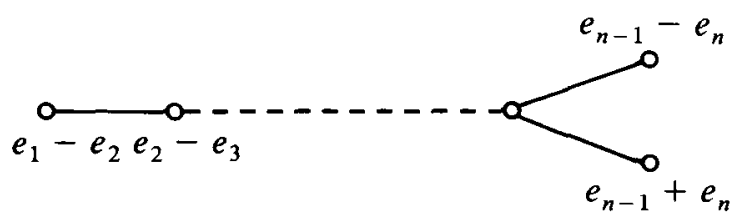

$\Phi\left(\mathbf{D}_{n}\right):$

$$
\left\{e_{i} \pm e_{j}, i \neq j, i, j=1,2, \ldots, n\right\}
$$

$\Pi\left(\mathbf{E}_{6}\right):$

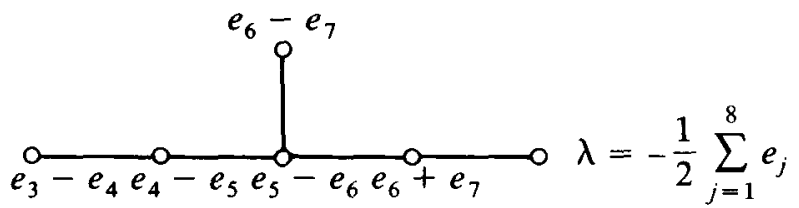

$\Phi\left(\mathbf{E}_{6}\right):$

$$
\begin{aligned}
& \left\{ \pm e_{i} \pm e_{j}, i \neq j, i, j=3,4,5,6,7,\right. \\
& \left. \pm\left(\frac{1}{2} \sum_{i=1}^{8} \varepsilon_{i} e_{i}\right), \varepsilon_{i}= \pm 1, \varepsilon_{1}=\varepsilon_{2}=\varepsilon_{8}=1, \prod_{i=1}^{8} \varepsilon_{i}=1\right\}
\end{aligned}
$$

$\Pi\left(\mathbf{E}_{7}\right)$ :

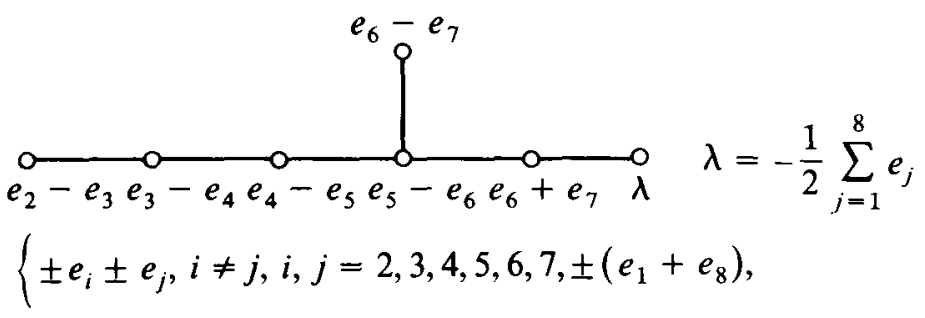

$$
\left.\pm\left(\frac{1}{2} \sum_{i=1}^{8} \varepsilon_{i} e_{i}\right), \varepsilon_{i}= \pm 1, \varepsilon_{1}=\varepsilon_{8}=1, \prod_{i=1}^{8} \varepsilon_{i}=1\right\}
$$




$$
\begin{aligned}
& \Pi\left(\mathbf{E}_{8}\right): \quad \overbrace{e_{1}-e_{2} e_{2}-e_{3} e_{3}-e_{4} e_{4}-e_{5} e_{5}-e_{6} e_{6}+e_{7} \lambda}^{\circ} \lambda=-\frac{1}{2} \sum_{j=1}^{8} e_{j} \\
& \Phi\left(\mathbf{E}_{8}\right): \quad\left\{ \pm e_{i} \pm e_{j}, i \neq j, i, j=1,2,3,4,5,6,7,8\right. \\
& \left. \pm\left(\frac{1}{2} \sum_{i=1}^{8} \varepsilon_{i} e_{i}\right), \varepsilon_{i}= \pm 1, \prod_{i=1}^{8} \varepsilon_{i}=1\right\}
\end{aligned}
$$

In this table, for each simple Lie algebra $L$ over $C$ (except $G_{2}$ and $F_{4}$ ), the simple root system $\Pi(L)$ and the root system $\Phi(L)$ which will be used in Table II are given.

\section{TABLE II}

In this table, for each Satake diagram $\Pi^{\theta}=(\Pi, \theta)$ (except the types given in Table $\left.\mathrm{II}^{\prime}\right), A_{\theta}=\left(\tau_{\theta} ; \Psi_{\theta} ; \varphi_{\theta}\right)$ defined in the proof of Proposition 1 are given.

Let $k$ be the number of the black nodes of the Satake diagram $\Pi^{\theta}$ and $n$ be the rank of $L$. We write $m=\frac{1}{2}(n-k)$ if $\Pi^{\theta}$ is of type AIII, $n=n-k$ if $\Pi^{\theta}$ is of the other types. Let $\varnothing$ denote the empty set.

\section{SATAKE Diagram}

AI

$$
\stackrel{\circ}{A_{\theta}=(1 ; \varnothing ; \varnothing)}
$$

Chevalley group

AII

$$
A_{\theta}=\left(I ; \varnothing ; e_{1}-e_{2}, e_{3}-e_{4}, \ldots, e_{n}-e_{n+1}\right)
$$

AIII

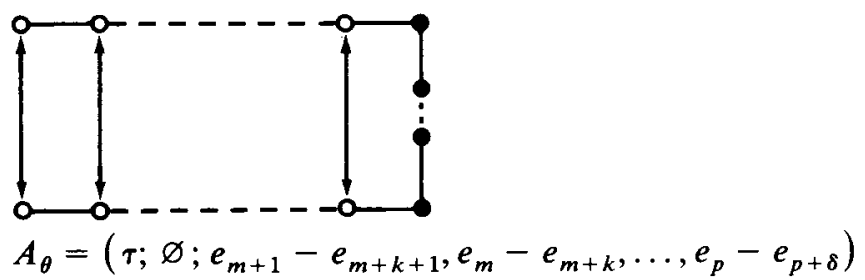

$p=\frac{1}{2} n$ and $\delta=2$ if $n$ is even, $p=\frac{1}{2}(n+1)$ and $\delta=1$ if $n$ is odd

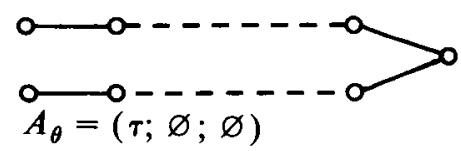

twisted group 
BI

$$
\begin{aligned}
& \stackrel{0}{A_{\theta}}=(I ; \varnothing ; \varnothing) \\
& \stackrel{0}{\circ}=\left(I ; e_{1}-e_{n}, e_{2}-e_{n}, \ldots, e_{m}-e_{n} ; e_{m+1}, e_{m+2}, \ldots, e_{n}\right) \text { if } k \text { is odd } \\
& A_{\theta}=\left(I ; \varnothing ; e_{m+1}, e_{m+2}, \ldots, e_{n}\right) \text { if } k \text { is even }
\end{aligned}
$$

CI

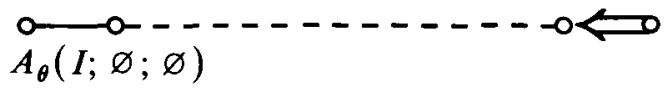

Chevalley group

CII

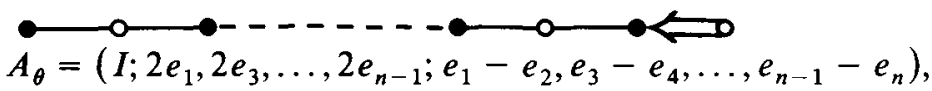

$n$ is even

DI

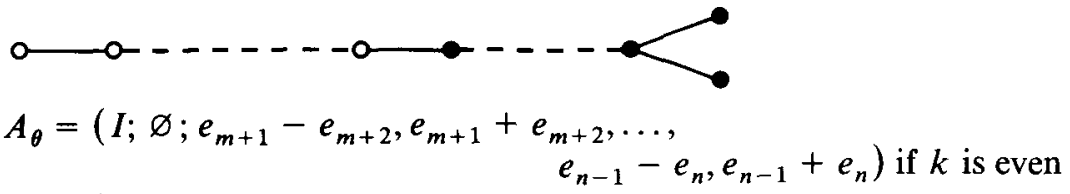
$A_{\theta}=\left(\tau ; \varnothing ; e_{m+1}-e_{m+2}, e_{m+1}+e_{m+2}, \ldots\right.$,

$$
\left.e_{n-2}-e_{n-1}, e_{n-2}+e_{n-1}\right) \text { if } k \text { is odd }
$$<smiles>Oc1ccco1</smiles>

twisted group

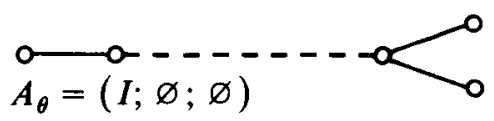

Chevalley group

DIII
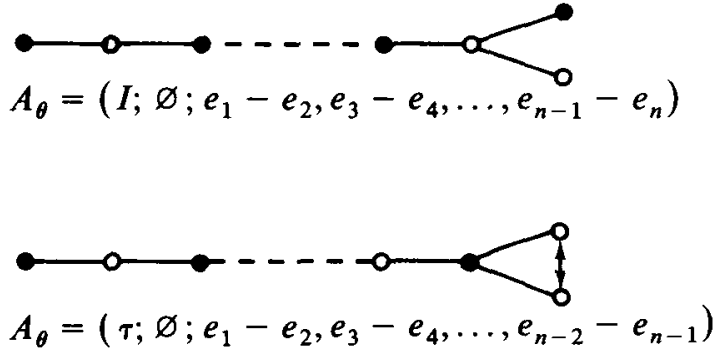
42

Cheng ChonHu

[30]

EI

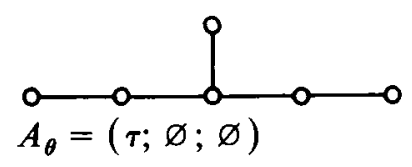

EII

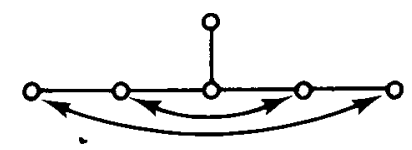

$$
A_{\theta}=(\tau ; \varnothing ; \varnothing)
$$

EIII

EIV

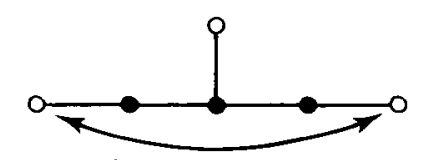

$$
A_{\theta}=\left(\tau ; \varnothing ; e_{4}+e_{7}, e_{5}-e_{6}\right)
$$

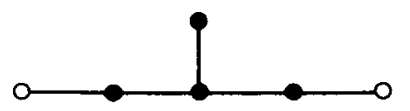

$$
A_{\theta}=\left(I ; \varnothing ; e_{4}-e_{5}, e_{4}+e_{5}, e_{6}-e_{7}, e_{6}+e_{7}\right)
$$

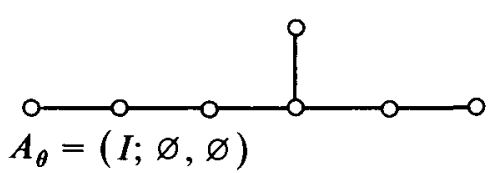

EVI

EVII

EVIII

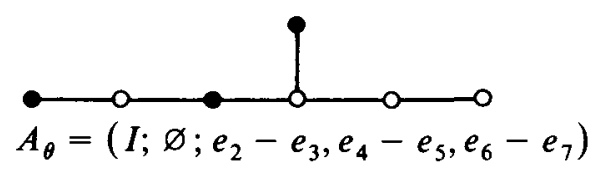

Chevalley group

twisted group

Chevalley group

Chevalley group

EIX
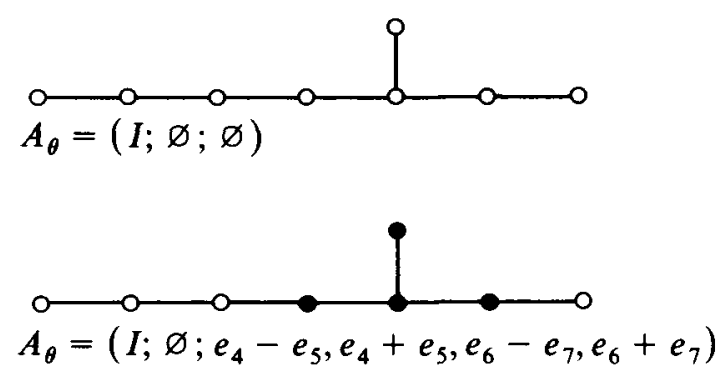

https://doi.org/10.1017/S1446788700028032 Published online by Cambridge University Press 
FI

$$
\stackrel{\circ}{\rightleftharpoons} \underset{A_{\theta}=(I ; \varnothing ; \varnothing)}{\rightleftharpoons}
$$

Chevalley group

G

$$
A_{\theta}=(I ; \varnothing ; \varnothing)
$$

Chevalley group

\section{TABLE II'}

CII

FII

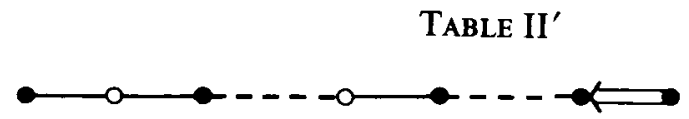

\section{References}

[1] R. W. Carter, Simple groups of Lie type, (Wiley, London, New York, 1972).

[2] Cheng ChonHu, 'Some simple groups of Lie type constructed by the inner automorphism', Chinese Ann. Math. 1 (1980), 161-176.

[3] C. Chevalley, 'Sur certain groupes simples', Tohoku Math. J. 7 (1955), 14-66.

[4] S. Helgason, Differential geometry, Lie groups, and symmetric spaces (Academic Press, New York, 1978).

[5] I. Satake, Classification theory of semisimple algebraic groups, (Dekker, New York, 1971).

[6] R. Steinberg, 'Variations on a theme of Chevalley', Pacific J. Math. 9 (1959), 875-891.

[7] J. Tits, 'Classification of algebraic semisimple groups', pp. 32-62 Proceedings of Symposia in Pure Math. Vol. 9, Algebraic groups and discontinous subgroups.

\section{Department of Mathematics}

Xiangtan University

Xiangtan, Hunan Province

Peoples' Republic of China 